\title{
A weighted corrective fuzzy reasoning spiking neural P system for fault diagnosis in power systems with variable topologies
}

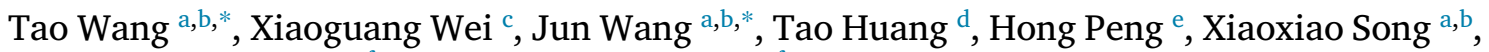 \\ Luis Valencia Cabrera ${ }^{\mathrm{f}}$, Mario J. Pérez-Jiménez ${ }^{\mathrm{f}}$ \\ ${ }^{a}$ Key Laboratory of Fluid and Power Machinery, Ministry of Education, Xihua University, Chengdu, 610039, China \\ b School of Electrical Engineering and Electronic Information, Xihua University, Chengdu, 610039, China \\ c School of Electrical Engineering, Southwest Jiaotong University, Chengdu, 610031, China \\ ${ }^{\mathrm{d}}$ Department of Energy, Politecnico di Torino, Torino 10129, Italy \\ e School of Computer and Software Engineering, Xihua University, Chengdu 610039, China \\ ${ }^{\mathrm{f}}$ Department of Computer Science and Artificial Intelligence, University of Sevilla, Avda. Reina Mercedes s/n, 41012, Spain
}

Keywords:

Fault diagnosis

Power system

Spiking neural P system

Fuzzy reasoning

Membrane computing

Cause-effect network

\begin{abstract}
A B S T R A C T
This paper focuses on power system fault diagnosis based on Weighted Corrective Fuzzy Reasoning Spiking Neural P Systems with real numbers (rWCFRSNPSs) to propose a graphic fault diagnosis method, called FDWCFRSNPS. In the FD-WCFRSNPS, an rWCFRSNPS is proposed to model the logical relationships between faults and potential warning messages triggered by the corresponding protective devices. In addition, a matrixbased reasoning algorithm for the rWCFRSNPS is devised to reason about the fault alarm messages using parallel representations. Besides, a layered modeling method based on rWCFRSNPSs is developed to adapt to topological changes in power systems and a Temporal Order Information Processing Method based on CauseEffect Networks is designed to correct fault alarm messages before the fault reasoning. Finally, in a case study considering a local subsystem of a $220 \mathrm{kV}$ power system, the diagnosis results of five test cases prove that the proposed FD-WCFRSNPS is viable and effective.
\end{abstract}

\section{Introduction}

One of the key goals when operating a power system is to reliably supply electric power to end users (Chen, 2011; Wei et al., 2017; Wang et al., 2019c). Unfortunately, faults are inevitable during power system operation because of a range of issues, such as natural, accidental and even malicious events (Huang et al., 2014; Bompard et al., 2016). When failures occur, the faulty areas are isolated immediately from the healthy parts of the system by protection relays, thus restricting the impact of the failures. To correctly carry out a black start strategy in the blackout area, it is critical to estimate which equipment is faulty. Thus, it is of great importance to have a good fault estimation method (Chen, 2011; Xiong et al., 2013b; Wang et al., 2015e).

In recent decades, various approaches to estimate faulty equipment have been proposed. These can be divided into three groups, based on the fault information is gathered from (1) remote signals, (2) remote measurements, or (3) multiple information sources. Methods in the first group estimate the faulty equipment using state variables, such as information about the operation of protective relays (PRs) and circuit breakers (CBs), or measurements of the temporal information or the start information of PRs from Supervisory Control And Data Acquisition (SCADA) systems. The second group instead considers electrical measurements (e.g., currents, voltages, the power, the phase or phase angles) from Wide Area Measurement Systems (WAMSs) with Phasor Measurement Units (PMUs). The last group uses both remote signals and measurements, occasionally including remote pulse volumes and control data. Of these three groups, the first has received the most attention, yielding practical implementations using, such as Expert Systems (ESs) (Lee et al., 2000; Ma et al., 2013; Vázquez et al., 1997), Artificial Neural Networks (ANNs) (Huang, 2002; Thukaram et al., 2005; Cardoso et al., 2008; Moghaddam et al., 2020), Optimization Methods (OMs) (Wang et al., 2015a; Lin et al., 2010; Wen and Han, 1995; Wang and Zhao, 2019), the Fuzzy Logic (FL) (Chang et al., 1997; Sun et al., 2004; Luo and Kezunovic, 2008; Chen, 2012; Chin, 2003; Zhou et al., 2020), Petri Nets (PNs) (Sun et al., 2004; Luo and Kezunovic, 2008; Yang et al., 2010; Mahmoudi-Nasr, 2019; Jiang et al., 2018), Bayesian Networks (BNs) (Chien et al., 2002; Zhu et al., 2006; Yang et al., 2019), Cause-Effect Networks (CENs) (Chen, 2011, 2012; Chen et al., 2011) and Spiking Neural P Systems (SNPSs) (Xiong et al., 2013b; Wang et al., 2015e,b; Peng et al., 2013; Xiong et al., 2013a). Each method has its own advantages and disadvantages, which are described in Wang et al. (2015e) and Luo and Kezunovic (2008).

\footnotetext{
* Corresponding authors at: School of Electrical Engineering and Electronic Information, Xihua University, Chengdu, 610039, China. E-mail addresses: wangatao2005@163.com (T. Wang),wj.xhu@hotmail.com (J. Wang).
} 
Among the methods, the SNPSs-based ones are graphical models based on Fuzzy Reasoning Spiking Neural P Systems (FRSNPSs) and are somewhat unusual in their ability to both handle uncertainty and process information in parallel, which is a characteristic that is very important for diagnosing faults in power systems. Given that, this paper focuses on an SNPS-based fault diagnosis method and its application to estimate faulty equipment in power systems. The SNPSs (Ionescu et al., 2006; Peng et al., 2019) are a new class of high performance distributed and parallel neural computing devices inspired by the behaviors of neurons. They send electrical impulses (spikes) along axons from presynaptic neurons to postsynaptic neurons, as shown in Fig. 1. Thus, an SNPS can further be represented by a directed graph, where nodes represent neurons, arcs are synapses indicating synaptic connections between neurons, and the information (spikes) flow along the arcs (synapses) (Díaz-Pernil et al., 2013; Zeng et al., 2014; Zhang et al., 2015). SNPSs aim to incorporate specific ideas from spiking neurons into membrane computing and essentially combine Spiking Neural Networks (SNNs) (Zhang et al., 2014) with P systems (Păun, 2000; Song et al., 2017; Cabarle et al., 2017). Recently, SNPSs have received significant attention, in terms of investigating both their theory (Pan et al., 2017, 2018; Wu et al., 2018; Díaz-Pernil and Gutiérrez-Naranjo, 2018) and applications (Wang et al., 2015b), where fault diagnosis is a very important application of SNPSs and is currently a hot topic that has yielded promising results.

To make SNPSs suitable for solving fault diagnosis problems, a Fuzzy Reasoning Spiking Neural P System with real numbers (rFRSNPS) (Peng et al., 2013) has been proposed within an SNPS and membrane computing framework, and a parallel fuzzy reasoning algorithm has been developed for fault knowledge representation and reasoning. The rFRSNPS introduced probabilities to describe the potential values of spikes and the fuzzy truth values of neurons. That study is the first attempt to perform fault diagnosis using SNPSs for a simple case involving only one transformer. Even though the model is simple, it provides a foundation for SNPSs-based fault diagnosis methods. Subsequently, an rFRSNPS was applied to diagnosing power system faults for the first time and was test on three example power systems to verify its effectiveness (Xiong et al., 2013b,a). After that, Adaptive Fuzzy Reasoning Spiking Neural P Systems (AFRSNPSs) (Wang and Peng, 2013) were also used to diagnose power system faults (Tu et al., 2014), demonstrating their ability to model weighted fuzzy production rules and showing that they can learn and adjust their weights automatically. At that time, SNPS-based fault diagnosis models were developed step-by-step, but they should really use historical statistics, which are frequently difficult to obtain and update in practical engineering.

Consequently, to handle the incompleteness and uncertainty inherent in the fault diagnosis of power systems and avoid historical data issues, an SNPS-based method called FDSNP (Wang et al., 2015e) was proposed. The FDSNP introduced a Fuzzy Reasoning Spiking Neural P System with trapezoidal fuzzy numbers (tFRSNPS) (Wang et al., 2014b) to model potentially faulty equipment. In addition, an algebraic fuzzy reasoning algorithm was designed for the tFRSNPS to obtain fault confidence levels of the potentially faulty equipment, and fault fuzzy production rule sets were designed to describe the relationships between faults and protection devices. For the first time, this gave us a clear algorithmic description of SNPS-based fault diagnosis methods and demonstrated the powerful ability of trapezoidal fuzzy numbers (Wang et al., 2015c) to deal with incomplete and uncertain fault information. This was crucial in promoting the study of SNPSsbased methods. Later, Intuitionistic Fuzzy Spiking Neural P Systems (IFSNPSs) (Peng et al., 2018) were developed by introducing intuitionistic fuzzy sets into the SNPS framework, and Fuzzy Reasoning Spiking Neural P Systems with interval-valued fuzzy numbers (ivFRSNPSs) (Yu et al., 2017; Wang et al., 2019a) were also proposed by combining interval-valued fuzzy numbers with SNPSs. To verify their effectiveness, both the IFSNPS and ivFRSNPS have been used to diagnose faults in a six-bus $69 \mathrm{kV}$ distribution system. The triangular Fuzzy Spiking Neural
P System (TFSNPS) (Tao et al., 2017), which integrates triangular fuzzy numbers into SNPSs, has also been proposed and used to create fault diagnosis models for high voltage networks to verify their feasibility and effectiveness in two example scenarios. The above methods deal the incompleteness and uncertainty in alarm messages efficiently; however, they all strongly dependent on historical statistics or expertise. Therefore, a fault diagnosis method for power transmission lines based on an spiking neural P system with self-updating rules (Liu et al., 2020) was proposed, where the attribute reduction capacity of rough sets and the apoptosis mechanism of neurons were integrated in a $\mathrm{P}$ system. Moreover, a new rough set-based bio-inspired fault diagnosis method (Wang et al., 2020) for electrical substations was proposed. The two methods can deal with uncertain and incomplete fault alarm messages without historical statistic and expert experience.

Besides, SNPSs have also been used to diagnose faults in other types of power systems. For example, the rFRSNPS has been applied to diagnose faults in electric locomotive systems (Wang et al., 2014a), describing the relationships between the breakdown signals and faulty equipment in subsystems by building fault diagnosis models. In addition, Weighted Fuzzy Reasoning Spiking Neural P Systems (WFRSNPSs) have been used to diagnose faults in traction power supply systems (TPSSs) of China high-speed railways (Wang et al., 2015d), where fault diagnosis production rules for TPSSs and corresponding WFRSNPS models were created. A Modified Fuzzy Reasoning Spiking Neural P System (MFRSNPS) (He et al., 2015) has also been proposed on the basis of both an rFRSNPS and a tFRSNPS, and was applied to fault diagnosis of metro traction power supply systems. Besides, a new framework for the fault propagation path modeling of power systems based on membrane computing was proposed in Wang et al. (2019b), where an event spiking neural $P$ system with neurotransmitter concentration was devised to intuitively reveal fault propagation paths using graphic models and parallel knowledge reasoning ability of SNPSs. That work innovatively extended the application of SNPSs from a single element to a system-wise investigation as well as from the post-ante application to a new ex-ante framework.

Each of these SNPS-based fault diagnosis methods has its own strengths and weaknesses. However, none of them consider temporal order information, which is very helpful for correcting remote signals from SCADA systems and hence improving the fault diagnosis results. Consequently, a method based on a temporal fuzzy reasoning spiking neural P system with real numbers (FDTSNP) was developed to diagnose power system faults (Huang et al., 2016), in which consistency constraints on the temporal order information were used to correct the alarm information. However, such constraint relationships are not easy to understand and implement in real systems. In addition, none of the aforementioned SNPS-based methods allow for the fault diagnosis models to be adaptively rebuilt when the topology of a power system changes, which is very important when operating power grids with large and complex workloads in practice. So, further work must be done to make best use of the temporal order information contained in remote signals in a simpler way and design SNPS-based models that can adapt to power system topological changes. Therefore, this paper proposes a graphical Fault Diagnosis method based on Weighted Corrective Fuzzy Reasoning Spiking Neural P Systems with real numbers (rWCFRSNPSs), called FD-WCFRSNPS. The main contributions of this paper are described as follows:

- Targeted to improve the correctness of fault diagnosis results by correcting alarm messages in an objective way, this study proposes a Temporal Order Information Processing Method based on Cause-Effect Networks (TOIPM-CEN). The TOIPM-CEN is a graphical method that can make the most of the temporal order information in an intuitive and intelligible way, making it easy to understand and implement. 


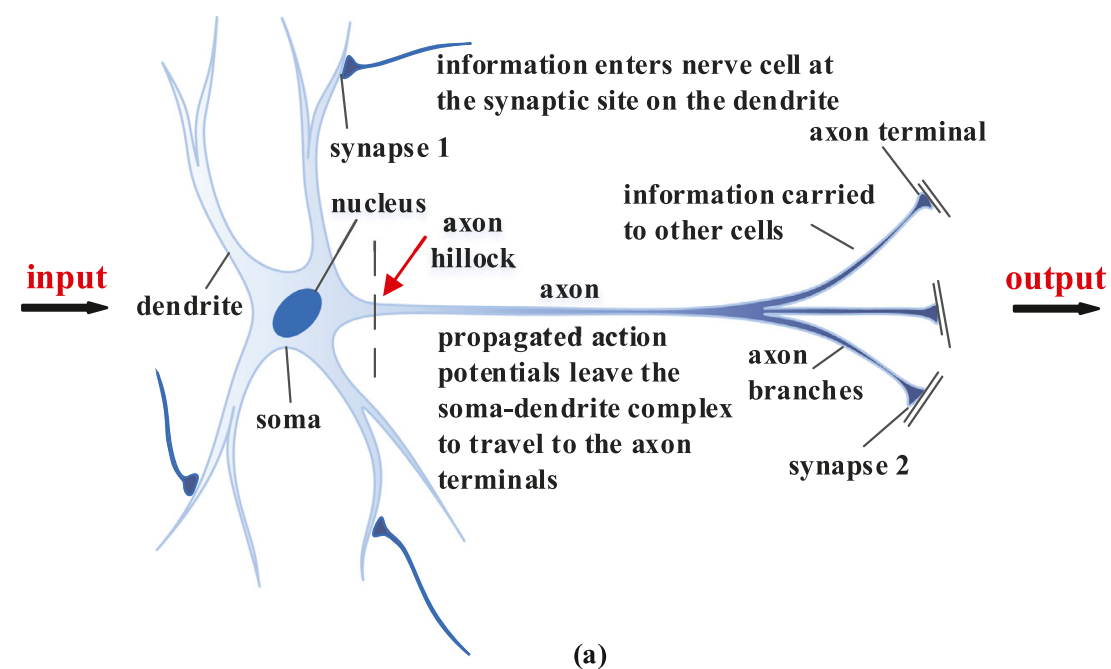

(a)

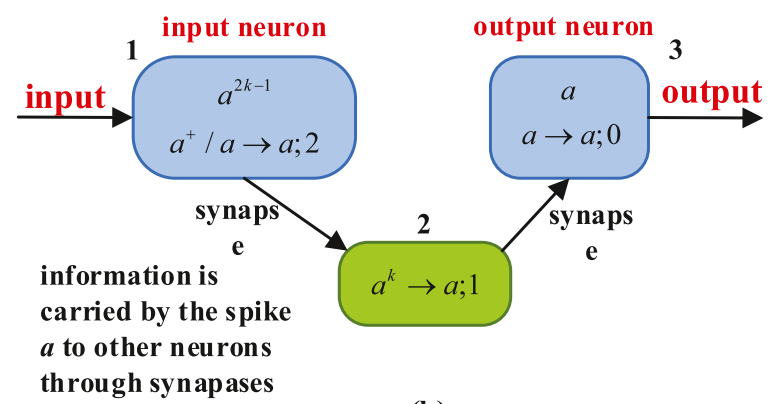

(b)

Fig. 1. Spiking neural P systems. (a) Illustration of a neuron. (b) A simple example of an SNPS.

- A layered modeling method based on rWCFRSNPSs is proposed. It means our rWCFRSNPS-based fault diagnosis models are able to adapt well to topological changes of power systems, which is a key strength in the new power environment of the energy internet that often involves changes in the topology.

- This paper proposes an rWCFRSNPS and its matrix-based reasoning algorithm to create the FD-WCFRSNPS, which offers an intuitive graphical illustration of the problem (based strictly on mathematical expressions), the ability to easily adapt diagnosis models to topological changes, and strong fault tolerance (supported by a parallel design, fault confidence levels in $[0,1]$ and the use of the temporal logic).

The remainder of this paper is organized as follows. Section 2 presents the rWCFRSNPS and its MBRA. Section 3 proposes the FDWCFRSNPS. Then, case studies are provided in Section 4. Finally, Section 5 presents our conclusions and future research directions.

\section{2. rWCFRSNPs}

First, we define an rWCFRSNPS with one type of proposition neurons and three types of rule neurons, describing the necessary syntax and semantics in detail. After introducing the model, we then describe the knowledge reasoning algorithm for the rWCFRSNPS, that is, the MBRA.

\subsection{Definitions}

Definition 1. A Weighted Corrective Fuzzy Reasoning Spiking Neural P System with real numbers (rWCFRSNPS) of degree $m \geq 1$ is a tuple

$\Pi=\left(O, \sigma_{1}, \ldots, \sigma_{m}\right.$, syn, in, out $)$

where:
(1) $O=\{a\}$ is a singleton alphabet ( $a$ is called spike);

(2) $Q=Q_{p} \cup Q_{r}$ is the complete neuron set, consisting of the proposition neuron set $Q_{p}=\left\{\sigma_{1}, \ldots, \sigma_{s}\right\}$ and the rule neuron set $Q_{r}=\left\{\sigma_{s+1}, \ldots, \sigma_{s+t}\right\}$, where $s+t=m$;

(3) $\operatorname{syn} \subseteq\{1,2, \ldots, m\} \times\{1,2, \ldots, m\}$ with $(i, i) \notin$ syn, for $1 \leq i \leq m$, is a directed graph of synapses between the linked neurons;

(4) in, out $\in\{1,2, \ldots, m\}$ indicate the input neuron set and the output neuron set of $\Pi$, respectively.

Each proposition neuron $\sigma_{i}(1 \leq i \leq s)$ is of the form $\left(\theta_{i}, t_{i}, \vec{\omega}_{i}, \lambda_{i}, r_{i}\right)$, where

(1) $\theta_{p i}$ is a real number in $[0,1]$ representing the potential value of the spikes (electrical impulses) contained in $\sigma_{i}$;

(2) $t_{i} \in\{0,1\}$ is the information correction parameter, which represents whether the information carried by the spike of proposition neuron $\sigma_{i}$ is valid $\left(t_{i}=1\right)$ or not $\left(t_{i}=0\right)$;

(3) $\vec{\omega}_{i}=\left(\omega_{i, 1}, \ldots, \omega_{i, M_{i}}\right)$ is a real number vector in $(0,1]$ representing the output weight vector of the proposition neuron $\sigma_{i}$, where $\omega_{i, k} \in(0,1]$, for $1 \leq k \leq M_{i}$, represents the weight of the $k$ th output arc (synapse) of proposition neuron $\sigma_{i}$ and $M_{i}$ is a natural number representing the number of pro-synaptic neurons of $\sigma_{i}$, that is, the number of synapses starting from $\sigma_{i}$;

(4) $\lambda_{i}$ is a real number in $[0,1)$ representing the firing threshold of proposition neuron $\sigma_{i}$

(5) $r_{i}$ represents the firing (spiking) rule of the proposition neuron $\sigma_{i}$ with the form $E / a^{\theta} \rightarrow a^{\beta}$, where $\theta$ and $\beta$ are real numbers in [0,1], and $E=\left\{a^{n}, \theta \geq \lambda_{i}\right\}$ is the firing condition.

Each rule neuron $\sigma_{s+j}(1 \leq j \leq t)$ is of the form $\left(\theta_{j}, c_{j}, \vec{\omega}_{j}, \lambda_{j}, r_{j}\right)$, where

(1) $\theta_{r j}$ is a real number in $[0,1]$ representing the potential value of spikes (electrical impulses) it contains; 


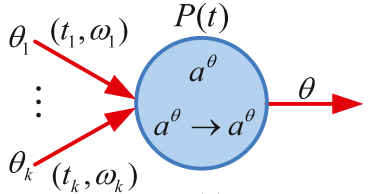

(a)

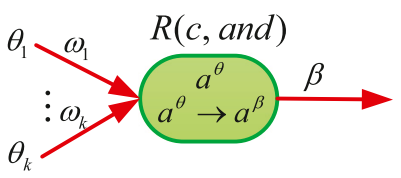

$\beta=\min \left\{\theta_{1} * \omega_{1}+\ldots+\theta_{k} * \omega_{k}\right\} * c$

(c)

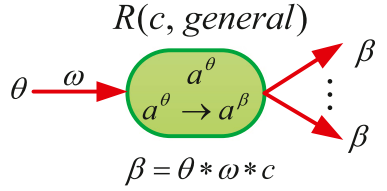

(b)

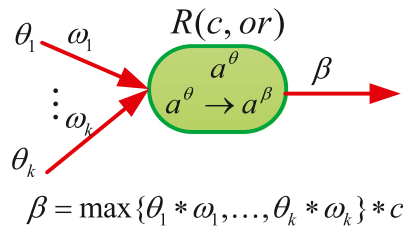

(d)
Fig. 2. Graphical representations of (a) proposition, (b) general rule, (c) and rule, and (d) or rule neurons.

(2) $c_{j}$ is a real number in $[0,1]$ representing its truth value, which is equivalent to the certainty factor of its associated fuzzy production rule;

(3) $\vec{\omega}_{j}=\left(\omega_{j, 1}, \ldots, \omega_{j, N_{j}}\right)$ is a real valued vector in $(0,1]$ representing its output weight vector, where $\omega_{j, k}\left(1 \leq k \leq N_{j}\right)$ represents the weight of its $k$ th output arc (synapse), and $N_{j}$ is a natural number representing the number of its prosynaptic neurons, that is, the number of synapses starting from $\sigma_{s+j}$;

(4) $\lambda_{j}$ is a real number in $[0,1)$ representing its firing threshold;

(5) $r_{j}$ represents its firing (spiking) rule in the form $E / a^{\theta} \rightarrow a^{\beta}$, where $\theta$ and $\beta$ are real numbers in [0,1], and $E=\left\{a^{n}, \theta \geq \lambda_{j}\right\}$ is the firing condition. The firing rule $r_{j}$ of the rule neuron $\sigma_{s+j}$ can be applied at instant $t$ if and only if it receives at least $n$ spikes at that moment with a potential value of $\theta \geq \lambda_{j}$. Applying rule $r_{j}$ consumes (remove) $n$ spikes and produces (emits) one spike;

The rWCFRSNPS involves four types of neurons, namely, proposition, general rule, and rule and or rule neurons, and are defined as follows. (These definitions are similar to those in Wang et al. (2015d), except for some parameters and the ways that spikes are processed in neurons.)

Proposition neurons are associated with propositions in fuzzy production rules. They are represented by circles and the symbol $P$, as shown in Fig. 2(a), and the corresponding simplified form is shown in Fig. 3(a). If the proposition neuron $\sigma_{i}=\left(\theta_{i}, t_{i}, \vec{\omega}_{i}, \lambda_{i}, r_{i}\right)$ is an input neuron, then its initial potential $\theta=\theta_{i} * t_{i}$, where $\theta_{i}$ is the information it receives from the environment, $t_{i}$ is the information correction parameter, and $\theta$ is the corrected results of environmental information received. Otherwise, $\theta$ is the result of applying a logical or operation to all the weighted potential values it receives from its presynaptic rule neurons; that is, $\theta=\max \left\{\theta_{1} * t_{i} * \vec{\omega}_{1}, \ldots, \theta_{k} * t_{i} * \vec{\omega}_{k}\right\}$, where $\theta_{1}, \ldots, \theta_{k}$ are the environmental potentials. The neuron's firing rule $r_{i}$ is of the form $E / a^{\theta} \rightarrow a^{\theta}$, where $E=\left\{a^{n}, \theta \geq \lambda_{i}\right\}$; that is, the parameter $\beta$ of the firing rule contained in such a neuron is identical to $\theta$. This rule can be applied at instant $t$ if and only if the neuron receives at least $n$ spikes at that moment with a potential $\theta \geq \lambda_{i}$. Applying the rule $r_{i}$ consumes the potential $\theta$ of spikes contained in the neuron and causes a new spike with potential $\theta$ to be produced and emitted.

General rule neurons are associated with fuzzy production rules that only have one proposition in their antecedents. They are represented by rounded rectangles and the symbol $R(c$, general $)$, as shown in Fig. 2(b), and the corresponding simplified form is shown in Fig. 3(b). They only have one presynaptic proposition neuron, together with one or more postsynaptic proposition neurons. If the general rule neuron $\sigma_{j}=\left(\theta_{j}, c_{j}, \vec{\omega}_{j}, \lambda_{j}, r_{j}\right)$ receives a spike from its presynaptic proposition neuron and its firing condition $E=\left\{a^{n}, \theta_{\geq} \lambda_{j}\right\}$ is satisfied, then it fires by applying the rule $E / a^{\theta} \rightarrow a^{\beta}$, producing a new spike with potential

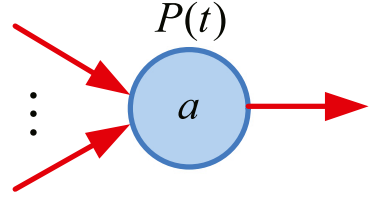

(a)

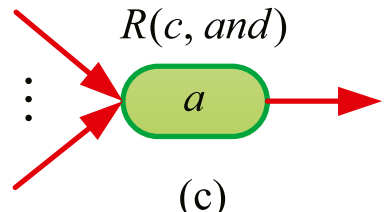

(c)

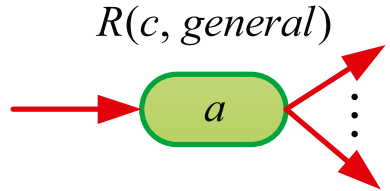

(b)

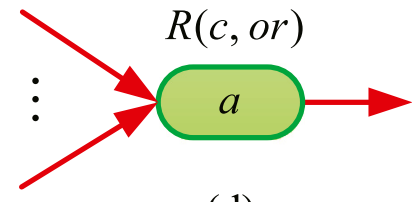

(d)
Fig. 3. Simplified representations of (a) proposition, (b) general rule, (c) and rule, and (d) or rule neuron.

$\beta=\theta_{1} * \vec{\omega}_{j} * c_{j}$. Note that the potential $\theta_{1}$ is produced and corrected by the presynaptic proposition neuron of the neuron $\sigma_{j}$ and $\theta=\theta_{1}$.

And rule neurons are associated with fuzzy production rules that have more than one proposition in their antecedents, in an and relationship. They are represented by rounded rectangles and the symbol $R(c$, and ), as shown in Fig. 2(c), and the corresponding simplified form is shown in Fig. 3(c). They have more than one presynaptic proposition neuron, but just one postsynaptic proposition neuron. If the and rule neuron $\sigma_{j}=\left(\theta_{j}, c_{j}, \vec{\omega}_{j}, \lambda_{j}, r_{j}\right)$ receives $k$ spikes from its $k$ presynaptic proposition neurons and its firing condition $E=\left\{a^{n}, \theta_{\geq} \lambda_{j}\right\}$ is satisfied, then it fires by applying the rule $E / a^{\theta} \rightarrow a^{\beta}$, producing a new spike with potential $\beta=\min \left\{\theta_{1} * \vec{\omega}_{1}, \ldots, \theta_{k} * \vec{\omega}_{k}\right\} * c_{j}$. Note that the potentials $\theta_{1}, \ldots, \theta_{k}$ are produced and corrected by the presynaptic proposition neurons of the neuron $\sigma_{j}$ and $\theta=\min \left\{\theta_{1} * \vec{\omega}_{1}, \ldots, \theta_{k} * \vec{\omega}_{k}\right\}$.

Or rule neurons are associated with fuzzy production rules that have more than one proposition in their antecedents, in an or relationship. They are represented by rounded rectangles and the symbol $R(c, o r)$, as shown in Fig. 2(d), and the corresponding simplified form is shown in Fig. 3(d). They have more than one presynaptic proposition neuron, but only one postsynaptic proposition neuron. If the or rule neuron $\sigma_{j}=$ $\left(\theta_{j}, c_{j}, \vec{\omega}_{j}, \lambda_{j}, r_{j}\right)$ receives $k$ spikes from its $k$ presynaptic proposition neurons and its firing condition $E=\left\{a^{n}, \theta_{\geq} \lambda_{j}\right\}$ is satisfied, then it fires by applying the rule $E / a^{\theta} \rightarrow a^{\beta}$, producing a new spike with potential $\beta=\max \left\{\theta_{1} * \vec{\omega}_{1}, \ldots, \theta_{k} * \vec{\omega}_{k}\right\} * c_{j}$. Note that the potentials $\theta_{1}, \ldots, \theta_{k}$ are produced and corrected by the presynaptic proposition neurons of the neuron $\sigma_{j}$ and $\theta=\max \left\{\theta_{1} * \vec{\omega}_{1}, \ldots, \theta_{k} * \vec{\omega}_{k}\right\}$.

\section{2. $M B R A$}

In order for rWCFRSNPSs to represent and reason about knowledge efficiently, we propose the MBRA in this subsection. First, we introduce several matrices and vectors, along with related multiplication operators and functions. Then, we explain the algorithm in detail. Throughout this subsection, the index $i(1 \leq i \leq s)$ refers to the $i$ th proposition neuron $\sigma_{i}$, whereas the index $j(1 \leq j \leq t)$ refers to the $j$ th rule neuron $\sigma_{s+j}$. Now, we set out some essential notation for what follows.

(1) $\boldsymbol{W}_{p}=\left(\omega_{j i}\right)_{t \times s}$ is a synaptic weight matrix representing the directed connections with weights from rule neurons to proposition neurons. Specifically, if there is a directed arc (synapse) from rule neuron $\sigma_{s+j}$ to proposition neuron $\sigma_{i}$, then $\omega_{j i}\left(\omega_{j i} \in(0,1]\right)$ is equal to the output weight of synapse $(j, i)$; otherwise, $\omega_{j i}=0$.

(2) $\boldsymbol{W}_{r 1}=\left(\omega_{i j}\right)_{s \times t}$ is a synaptic weight matrix representing the directed connections with weights from proposition neurons to general rule neurons. Specifically, if there is a directed arc (synapse) from proposition neuron $\sigma_{i}$ to general rule neuron $\sigma_{s+j}$, then $\omega_{i j}$ $\left(\omega_{i j} \in(0,1]\right)$ is equal to the output weight of synapse $(i, j)$; otherwise, $\omega_{i j}=0$. 
(3) $\boldsymbol{W}_{r 2}=\left(\omega_{i j}\right)_{s \times t}$ is a synaptic weight matrix representing the directed connections with weights from proposition neurons and and rule neurons. Specifically, if there is a directed arc (synapse) from proposition neuron $\sigma_{i}$ to and rule neuron $\sigma_{s+j}$, then $\omega_{i j}\left(\omega_{i j} \in\right.$ $(0,1])$ is equal to the output weight of synapse $(i, j)$; otherwise, $\omega_{i j}$ $=0$.

(4) $\boldsymbol{W}_{r 3}=\left(\omega_{i j}\right)_{s \times t}$ is a synaptic weight matrix representing the directed connections with weights from proposition neurons to or rule neurons. Specifically, if there is a directed arc (synapse) from proposition neuron $\sigma_{i}$ to or rule neuron $\sigma_{s+j}$, then $\omega_{i j}\left(\omega_{i j} \in(0,1]\right)$ is equal to the output weight of synapse $(i, j)$; otherwise, $\omega_{i j}=0$.

(5) $\boldsymbol{D}_{1}=\left(d_{i j}\right)_{s \times t}$ is a binary matrix representing the directed connections from proposition neurons to rule neurons. Specifically, if there is a directed arc (synapse) from proposition neuron $\sigma_{i}$ to rule neuron $\sigma_{s+j}$, then $d_{i j}=1$; otherwise, $d_{i j}=0$.

(6) $\boldsymbol{D}_{2}=\left(d_{j i}\right)_{t \times s}$ is a binary matrix representing the directed connections from rule neurons to proposition neurons. Specifically, if there is a directed arc (synapse) from rule neuron $\sigma_{s+j}$ to proposition neuron $\sigma_{i}$, then $d_{j i}=1$; otherwise, $d_{j i}=0$.

(7) $\boldsymbol{C}=\operatorname{diag}\left(c_{1}, \ldots, c_{t}\right)$ is a diagonal matrix, where $c_{j}(1 \leq j \leq t)$ is a real number in $[0,1]$ representing the certainty factor of the $j$ th rule neuron $\sigma_{s+j} . \square$

(8) $\boldsymbol{T}=\operatorname{diag}\left(t_{1}, \ldots, t_{s}\right)$ is a diagonal matrix, called the information correction parameters matrix, where $t_{i}(1 \leq i \leq s)$ is a real Boolean number in $\{0,1\}$ representing the information correction parameter of the $i$ th proposition neuron $\sigma_{i}$, indicating whether the information carried by its spike is reliable $\left(t_{i}=1\right)$ or not $\left(t_{i}=0\right)$.

(9) $\lambda_{p}=\left(\lambda_{p 1}, \ldots, \lambda_{p s}\right)^{T}$ is the threshold vector for the $s$ proposition neurons, where $\lambda_{p i}(1 \leq i \leq s)$ is a real number in $[0,1)$ representing the firing threshold of the $i$ th proposition neuron $\sigma_{i}$.

(10) $\lambda_{r}=\left(\lambda_{r 1}, \ldots, \lambda_{r t}\right)^{T}$ is the threshold vector for the $t$ rule neurons, where $\lambda_{r j}(1 \leq j \leq t)$ is a real number in $[0,1)$ representing the firing threshold of the $j$ th rule neuron $\sigma_{s+j}$.

(11) $\theta_{p}=\left(\theta_{p 1}, \ldots, \theta_{p s}\right)^{T}$ is the (real-valued) truth vector for the $s$ proposition neurons, where $\theta_{p i}(1 \leq i \leq s)$ is a real number in $[0,1]$ representing the spike value of the $i$ th proposition neuron $\sigma_{i}$. If a proposition neuron does not contain any spikes, its spike value is 0 .

(12) $\theta_{r}=\left(\theta_{r 1}, \ldots, \theta_{r s}\right)^{T}$ is the (real-valued) truth vector for the $t$ rule neurons, where $\theta_{r j}(1 \leq j \leq t)$ is a real number in $[0,1]$ representing the spike value of the $j$ th proposition neuron $\sigma_{s+j}$. Again, if a proposition neuron does not contain any spikes, its spike value is 0 .

(13) $a_{p}=\left(a_{p 1}, \ldots, a_{p s}\right)^{T}$ is the spike number vector for the $s$ proposition neurons, where $a_{p i}(1 \leq i \leq s)$ is an integer number representing the number of spikes in the $i$ th proposition neuron $\sigma_{i}$.

(14) $a_{r}=\left(a_{r 1}, \ldots, a_{r t}\right)^{T}$ is the spike number vector for the $t$ rule neurons, where $a_{r j}(1 \leq j \leq t)$ is an integer number representing the number of spikes in the $j$ th rule neuron $\sigma_{s+j}$.

(15) $\boldsymbol{\beta}_{p}=\left(\beta_{p 1}, \ldots, \beta_{p s}\right)^{T}$ is the (real-valued) output spike value vector for the $s$ proposition neurons before correction, where $\beta_{p i}(1 \leq$ $1 \leq s)$ is a real number in $[0,1]$ representing the output spike value of the $i$ th proposition neuron $\sigma_{i}$ after it fires.

(16) $\beta_{r}=\left(\beta_{r 1}, \ldots, \beta_{r t}\right)^{T}$ is the (real-valued) output spike value vector for the $t$ rule neurons before correction, where $\beta_{r j}(1 \leq j \leq t)$ is a real number in $[0,1]$ representing the output spike value of the $j$ th proposition neuron $\sigma_{s+j}$ after it fires.

(17) $\boldsymbol{b}_{p}=\left(b_{p 1}, \ldots, b_{p s}\right)^{T}$ is the output spike number vector for the $s$ proposition neurons after correction, where $b_{p i}(1 \leq i \leq s)$ is an integer number representing the output spike number of the $i$ th proposition neuron $\sigma_{i}$ after it fires.
(18) $\boldsymbol{b}_{r}=\left(b_{r 1}, \ldots, b_{r t}\right)^{T}$ is the output spike number vector for the $t$ rule neurons after correction, where $b_{r j}(1 \leq j \leq t)$ is an integer number representing the output spike number of the $j$ th rule neuron $\sigma_{s+j}$ after it fires.

Next, we define some multiplication operators and functions relating to the above matrices and vectors.

(1) Multiplication operator $\otimes$ :

$$
\boldsymbol{W}_{r 1}^{T} \otimes \boldsymbol{\theta}_{p}=\left(\bar{\omega}_{1}, \ldots, \bar{\omega}_{t}\right)^{T}
$$

where $\bar{\omega}_{j}=\omega_{i j} * \theta_{p i}$, for $1 \leq i \leq s, 1 \leq j \leq t$. Eq. (2) is used to calculate the spike values of general rule neurons.

(2) Multiplication operator $\oplus$ :

$\boldsymbol{W}_{r 2}^{T} \oplus \boldsymbol{\theta}_{p}=\left(\bar{\omega}_{1}, \ldots, \bar{\omega}_{t}\right)^{T}$

where $\bar{\omega}_{j}=\min \left\{\omega_{i j} * \theta_{p i} \mid 1 \leq i \leq s\right\}$, for $1 \leq j \leq t$. Eq. (3) is used to calculate the spike values of and rule neurons.

(3) Multiplication operator $\odot$ :

$\boldsymbol{W}_{r 3}^{T} \odot \boldsymbol{\theta}_{p}=\left(\bar{\omega}_{1}, \ldots, \bar{\omega}_{t}\right)^{T}$

where $\bar{\omega}_{j}=\max \left\{\omega_{i j} * \theta_{p i} \mid 1 \leq i \leq s\right\}$, for $1 \leq j \leq t$. Eq. (4) is used to calculate the spike values of or rule neurons.

or

$\boldsymbol{W}_{p}^{T} \odot \boldsymbol{\theta}_{\boldsymbol{r}}=\left(\bar{\omega}_{1}, \ldots, \bar{\omega}_{s}\right)^{T}$

where $\bar{\omega}_{i}=\max \left\{\omega_{j i} * \theta_{r j} \mid 1 \leq j \leq t\right\}$, for $1 \leq i \leq s$. Eq. (5) is used to calculate the spike values of proposition neurons.

(4) Firing function of spike values: $\beta_{l}=\operatorname{fire}\left(\boldsymbol{\theta}_{l}, \boldsymbol{a}_{l}, \lambda_{l}\right)$ is defined as follows for $l \in\{p, r\}$

$\beta_{l k}=\left\{\begin{aligned} \theta_{l k}, & \text { if } a_{l k}=\lambda_{k}, \\ 0, & \text { if } a_{l k}<\lambda_{k},\end{aligned}\right.$

where $\beta_{l}=\left(\beta_{l 1}, \ldots, \beta_{l f}\right)^{T}, \theta_{l}=\left(\theta_{l 1}, \ldots, \theta_{l f}\right)^{T}, \boldsymbol{a}_{l}=\left(a_{l 1}, \ldots, a_{l f}\right)^{T}$, $\lambda_{l}=\left(\lambda_{l 1}, \ldots, \lambda_{l f}\right)^{T}, 1 \leq k \leq f$ with $f \in\{s, t\}$. Eq. (6) is the spike value firing function for proportion neurons (when $l=p$ ) or rule neurons (when $l=r$ ). It is employed to calculate the output spike values of corresponding neurons when the neurons fire.

(5) Firing function of spike numbers: $\boldsymbol{b}_{\boldsymbol{l}}=\operatorname{fire}\left(1, \boldsymbol{a}_{\boldsymbol{l}}, \lambda_{\boldsymbol{l}}\right)$ is defined as follows for $l \in\{p, r\}$

$b_{l k}=\left\{\begin{array}{l}1, \text { if } a_{l k}=\lambda_{k}, \\ 0, \text { if } a_{l k}<\lambda_{k},\end{array}\right.$

where $\boldsymbol{b}_{l}=\left(b_{l 1}, \ldots, b_{l f}\right)^{T}, \boldsymbol{a}_{l}=\left(a_{l 1}, \ldots, a_{l f}\right)^{T}, \lambda_{l}=\left(\lambda_{l 1}, \ldots, \lambda_{l f}\right)^{T}$, $1 \leq k \leq f$ with $f \in\{s, t\}$. Eq. (7) is the spike number firing function for proportion neurons (when $l=p$ ) or rule neurons (when $l=r$ ). It is employed to calculate the output spike numbers of corresponding neurons when the neurons fire.

(6) Update function of spike values: $\boldsymbol{\beta}_{l}=\operatorname{update}\left(\boldsymbol{\theta}_{l}, \boldsymbol{a}_{\boldsymbol{l}}, \lambda_{l}\right)$ is defined as follows for $l \in\{p, r\}$

$\beta_{l k}=\left\{\begin{array}{c}\beta_{l k}+\theta_{l k}, \text { if } 0<a_{l k}<\lambda_{l k}, \\ 0, \text { if } a_{l k}=0 \text { or } a_{l k}=\lambda_{l k},\end{array}\right.$

where $\beta_{l}=\left(\beta_{l 1}, \ldots, \beta_{l f}\right)^{T}, \theta_{l}=\left(\theta_{l 1}, \ldots, \theta_{l f}\right)^{T}, a_{l}=\left(a_{l 1}, \ldots, a_{l f}\right)^{T}$, $\lambda_{l}=\left(\lambda_{l 1}, \ldots, \lambda_{l f}\right)^{T}, 1 \leq k \leq f$ with $f \in\{s, t\}$. Eq. (8) is the update function for the spike values in proportion neurons (when $l=p$ ) or rule neurons (when $l=r$ ). It is employed to accumulate the spike values of corresponding neurons when the neurons do not fire.

(7) Update function of spike numbers: $\boldsymbol{a}=\operatorname{update}(\boldsymbol{e}, \boldsymbol{a}, \lambda)$ is defined as follows

$a_{k}=\left\{\begin{array}{c}e_{k}+a_{k}, \text { if } 0<a_{k}<\lambda_{k}, \\ 0, \text { if } a_{k}=0 \text { or } a_{k}=\lambda_{k},\end{array}\right.$

where $\boldsymbol{a}=\left(a_{1}, \ldots, a_{f}\right)^{T}, \boldsymbol{e}=\left(e_{1}, \ldots, e_{f}\right)^{T}, \lambda_{l}=\left(\lambda_{1}, \ldots, \lambda_{f}\right)^{T}$, $1 \leq k \leq f$ with $f \in\{s, t\} . e$ is a spike number factor representing 
the spike numbers that already exist in the neurons before new spikes come. Eq. (9) is the update function for the spike numbers in proportion neurons (when $f=s$ ) or rule neurons (when $f=$ $t$ ). It is employed to update the spike numbers of corresponding neurons when the neurons do not fire.

(8) Matrix $\boldsymbol{U}=\operatorname{diag}(b)$ (order $f \times f$ ): for $1 \leq k, q \leq f$, with $f \in\{s, t\}$ and matrix elements $\left(u_{k q}\right)_{f \times f}$,

$u_{k j}=\left\{\begin{array}{r}b_{k}, \text { if } k=q, \\ 0, \text { if } k \neq q,\end{array}\right.$

where $\boldsymbol{b}=\left(b_{1}, \ldots, b_{f}\right)$. Eq. (10) is employed to calculate the values of elements in the diagonal matrix evolved from the matrix $b$.

In what follows, we give a step-by-step description of the MBRA.

\section{MBRA}

INPUT: $\mathbf{W}_{p}, \mathbf{W}_{r 1}, \mathbf{W}_{r 2}, \mathbf{W}_{r 3}, \mathbf{D}_{1}, \mathbf{D}_{2}, \mathbf{C}, \mathbf{T}, \lambda_{p}, \lambda_{r}, \theta_{p}^{0}, a_{p}^{0}$. OUTPUT: Fuzzy truth values of output neurons.

Step 1: Set initial values and termination conditions

$\mathbf{0}_{1}=(0, \ldots, 0)_{s}^{T}$ and $\mathbf{0}_{2}=(0, \ldots, 0)_{t}^{T}$;

Step 2: Let $\boldsymbol{\theta}_{r}^{0}=(0, \ldots, 0)_{t}^{T}$ and $\boldsymbol{a}_{r}^{0}=(0, \ldots, 0)_{t}^{T}$;

Step 3: Let $g=0$;

Step 4: Process proposition neurons and rule neurons as follows:

(1) Process the states of proposition neurons. For each proposition neuron $p$ do the following:

$$
\left\{\begin{array}{l}
\boldsymbol{\beta}_{p}^{g}=\operatorname{fire}\left(\boldsymbol{\theta}_{p}^{g}, \boldsymbol{a}_{p}^{g}, \lambda_{p}\right) \\
\boldsymbol{b}_{p}^{g}=\operatorname{fire}\left(1, \boldsymbol{a}_{p}^{g}, \lambda_{p}\right) \\
\boldsymbol{\theta}_{p}^{g}=\operatorname{update}\left(\boldsymbol{\theta}_{p}^{g}, \boldsymbol{a}_{p}^{g}, \lambda_{p}\right) \\
\boldsymbol{a}_{p}^{g}=\operatorname{update}\left(\boldsymbol{a}_{p}^{g}, \boldsymbol{a}_{p}^{g}, \lambda_{p}\right) \\
\boldsymbol{b}_{p}^{g}=\operatorname{diag}\left(\boldsymbol{b}_{p}^{g}\right)
\end{array}\right.
$$

(2) Compute the spike values $\theta_{r}^{g+1}$ and number of spikes received $\boldsymbol{a}_{r}^{g+1}$ for rule neurons. For each rule neuron do the following:

$$
\boldsymbol{\theta}_{r}^{g+1}=\boldsymbol{W}_{r 1}^{T} \otimes\left[\left(\beta_{p}^{g} \otimes T\right)^{T}\right]+W_{r 2}^{T} \otimes\left[\left(\beta_{p}^{g} \otimes T\right)^{T}\right]+W_{r 3}^{T} \otimes\left[\left(\beta_{p}^{g} \otimes T\right)^{T}\right]
$$

$$
\boldsymbol{a}_{r}^{g+1}=\boldsymbol{a}_{r}^{g}+\left[\left(\boldsymbol{b}_{p}^{g} \oplus \boldsymbol{D}_{1}\right)^{T} \otimes \boldsymbol{b}_{p}^{g}\right] ;
$$

(3) Process the states of rule neurons. For each rule neuron and each proposition neuron do the following:

$$
\left\{\begin{array}{l}
\boldsymbol{\beta}_{r}^{g+1}=\operatorname{fire}\left(\boldsymbol{C} \otimes \boldsymbol{\theta}_{r}^{g+1}, \boldsymbol{a}_{r}^{g+1}, \lambda_{r}\right) \\
\boldsymbol{b}_{r}^{g+1}=\operatorname{fire}\left(1, \boldsymbol{a}_{r}^{g+1}, \lambda_{r}\right) \\
\boldsymbol{\theta}_{r}^{g+1}=\operatorname{update}\left(\boldsymbol{\theta}_{r}^{g+1}, \boldsymbol{a}_{p}^{g}, \lambda_{p}\right) \\
\boldsymbol{a}_{r}^{g+1}=\operatorname{update}\left(\boldsymbol{a}_{r}^{g+1}, \boldsymbol{a}_{p}^{g}, \lambda_{p}\right) \\
\boldsymbol{b}_{r}^{g+1}=\operatorname{diag}\left(\boldsymbol{b}_{r}^{g+1}\right)
\end{array}\right.
$$

(4) Compute the spike values $\theta_{p}^{g+1}$ and number of spikes received $\boldsymbol{a}_{p}^{g+1}$ for proposition neurons. For each rule neuron and each proposition neuron do the following:

$$
\begin{aligned}
& \theta_{p}^{g+1}=W_{p}^{T} \odot \boldsymbol{\beta}_{r}^{g+1} \\
& \boldsymbol{a}_{p}^{g+1}=\boldsymbol{a}_{p}^{g}+\left[\left(\boldsymbol{D}_{2} \oplus \boldsymbol{B}_{r}^{g+1}\right) \otimes \boldsymbol{b}_{r}^{g+1}\right.
\end{aligned}
$$

Step 5: If the termination conditions are satisfied, that is, $\boldsymbol{\theta}_{p}^{g+1}=\mathbf{0}_{1}$ and $\theta_{r}^{g+1}=\mathbf{0}_{2}$, then halt and export reasoning results, i.e., the fuzzy truth values of output neurons. Otherwise, $g=g+1$ and go to Step 4 .

\section{FD-WCFRSNPS}

In this section, we propose a graphical modeling approach, called FD-WCFRSNPS, for diagnosing power system faults using rWCFRSNPSs. First, we model fuzzy production rules based on rWCFRSNPSs, and then we design layered fault diagnosis models for equipment such as transmission lines, buses and transformers. Next, we adapt the method to process temporal order information. Finally, the FD-WCFRSNPS is algorithmically illustrated.

\subsection{Models for fuzzy production rules}

When diagnosing power system faults, the fault knowledge (alarm messages) is usually expressed as fuzzy production rules. In general, four types of rules are considered for fault diagnosis. In order to model them, we need to map them onto rWCFRSNPSs, as shown in Fig. 4. The rules and associated models can be described as follows.

Type 1 (Simple Rules) $R_{i}\left(\mathrm{CF}=c_{i}\right)$ : IF $p_{j}\left(\theta_{j}\right)$ THEN $p_{k}\left(\theta_{k}\right)$. The corresponding rWCFRSNPS is shown in Fig. 4(a), where $p_{j}$ and $p_{k}$ represent the rule's antecedent and consequent propositions, respectively, $c_{i}$ is a real number in $[0,1]$ representing the rule's certainty factor, and $\theta_{j}$ and $\theta_{k}$ are real numbers in $[0,1]$ representing the truth values of $p_{j}$ and $p_{k}$, respectively. The weight of proposition $p_{j}$ is $\omega_{j}$, where $\omega_{j}=1$ because there is only one antecedent proposition, and $t_{j}$ is the information correction parameter (either 0 or 1 ) of the proposition neuron $\sigma_{j}$. Thus, the truth value of $p_{k}$ is $\theta_{k}=\theta_{j} * t_{j} * \omega_{j} * c_{i}=\theta_{j} * t_{j} * c_{i}$.

Type 2 (Compound And Rules) ( $\mathrm{CF}=c_{i}$ ) $R_{i}$ : IF $p_{1}\left(\theta_{1}\right)$ AND ... AND $p_{k-1}\left(\theta_{k-1}\right)$ THEN $p_{k}\left(\theta_{k}\right)$. The corresponding rWCFRSNPS is shown in Fig. 4(b), where $p_{1}, \ldots, p_{k-1}$ and $p_{k}$ represent the rule's antecedent and consequent propositions, respectively, $c_{i}$ is a real number in [0, $1]$ representing the rule's certainty factor, and $\theta_{1}, \ldots, \theta_{k}$ are real numbers in $[0,1]$ representing the truth values of propositions $p_{1}, \ldots, p_{k}$, respectively. The weights of propositions $p_{1}, \ldots, p_{k-1}$ are $\omega_{1}, \ldots, \omega_{k-1}$, respectively, and $t_{1}, \ldots, t_{k-1}$ are information correction parameters (either 0 or 1 ) for the proposition neurons $\sigma_{1}, \ldots, \sigma_{k-1}$. Thus, the truth value of $p_{k}$ is $\theta_{k}=\min \left\{\theta_{1} * t_{1} * \omega_{1}, \ldots, \theta_{k-1} * t_{k-1} * \omega_{k-1}\right\} * c_{i}$.

Type 3 (Conclusion And Rules) ( $\left.\mathrm{CF}=c_{i}\right) R_{i}$ : IF $p_{1}\left(\theta_{1}\right)$ THEN $p_{2}\left(\theta_{2}\right)$ AND ... AND $p_{k}\left(\theta_{k}\right)$. The corresponding rWCFRSNPS is shown in Fig. 4(c), where $p_{1}$ and $p_{2}, \ldots, p_{k}$ represent the rule's antecedent and consequent propositions, $c_{i}$ is a real number in $[0,1]$ representing the rules' certainty factor, and $\theta_{1}, \ldots, \theta_{k}$ are real numbers in $[0,1]$ representing the truth values of propositions $p_{1}, \ldots, p_{k}$, respectively. The weight of proposition $p_{1}$ is $\omega_{1}$, where $\omega_{1}=1$ because there is only one antecedent proposition, and $t_{1}$ is the information correction parameter (either 0 or 1 ) for proposition neuron $\sigma_{1}$. This type of rule can be split into $k-1$ simple rules, so the truth values of $p_{2}, \ldots, p_{k}$ are $\theta_{2}=\cdots=\theta_{k}=\theta_{1} * t_{1} * \omega_{1} * c_{i}=\theta_{1} * t_{1} * c_{i}$.

Type 4 (Compound Or Rules) ( $\mathrm{CF}=c_{i}$ ) $R_{i}$ : IF $p_{1}\left(\theta_{1}\right)$ OR $\ldots$ OR $p_{k-1}\left(\theta_{k-1}\right)$ THEN $p_{k}\left(\theta_{k}\right)$. The corresponding rWCFRSNPS is shown in Fig. 4(d), where $p_{1}, \ldots, p_{k-1}$ and $p_{k}$ represent the rule's antecedent and consequent propositions, respectively, $c_{i}$ is a real number in $[0,1]$ representing the rule's certainty factor, and $\theta_{1}, \ldots, \theta_{k}$ are real numbers in $[0,1]$ representing the truth values of the propositions $p_{1}, \ldots, p_{k}$, respectively. The weights of propositions $p_{1}, \ldots, p_{k-1}$ are $\omega_{1}, \ldots, \omega_{k-1}$, respectively, and $t_{1}, \ldots, t_{k-1}$ are information correction parameters (either 0 or 1 ) for the proposition neurons $\sigma_{1}, \ldots, \sigma_{k-1}$. Thus, the truth value of $p_{k}$ is $\theta_{k}=\max \left\{\theta_{1} * t_{1} * \omega_{1}, \ldots, \theta_{k-1} * t_{k-1} * \omega_{k-1}\right\} * c_{i}$. 


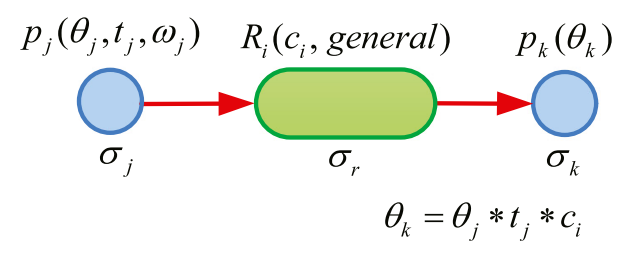

(a)

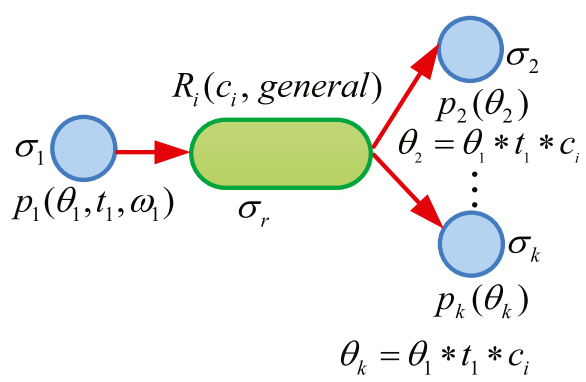

(c)

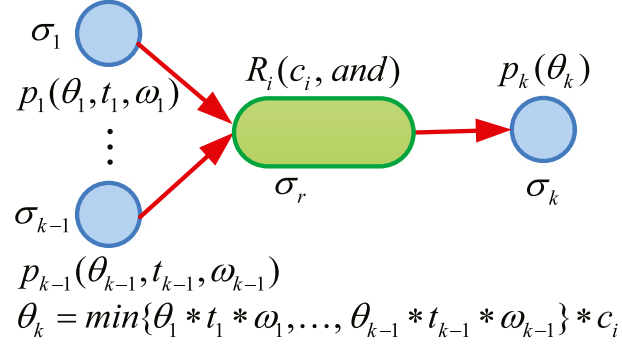

(b)

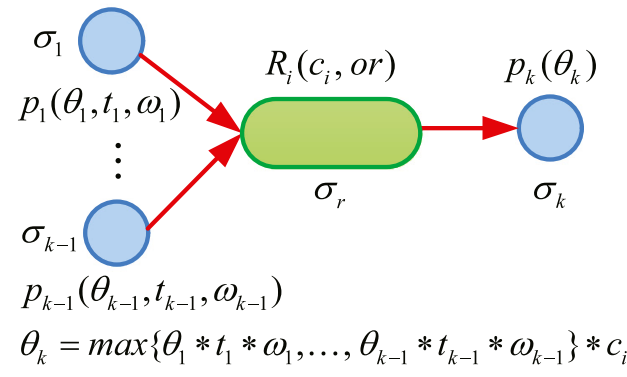

(d)

Fig. 4. rWCFRSNPSs for fuzzy production rules of (a) Type 1, (b) Type 2, (c) Type 3 and (d) Type 4.

\subsection{Layered fault diagnosis models and parameter values}

Here, we first propose layered fault diagnosis models based on rWCFRSNPSs for power system equipment such as transmission lines, buses and transformers. Then, we give adaptive adjustment rules to enable these models to adapt to changes in power system topology. Finally, suitable parameter values are discussed for the models.

\subsubsection{Layered models based on rWCFRSNPSs}

The fuzzy production rules discussed above can be used to describe the logical relationships between the information from protective devices and faults. We employ rWCFRSNPSs to model these rules, representing and reasoning about fault knowledge to find power system faults.

To improve the ability of SNPS-based fault diagnosis models to adapt to system topological changes, we propose layered fault diagnosis models based on rWCFRSNPSs for transmission lines, buses and transformers, as follows.

(1) Transmission lines

Because transmission lines include protective relays at both the sending and receiving ends, our fault diagnosis models for them should include separate submodels for both the sending and receiving ends. Given the way that protective relays operate, our models should also include one that integrates both ends, as well as sending end protection submodels including main, primary backup and secondary backup protection submodels, and similar submodels for the receiving end.

Fig. 5 illustrates the complete transmission line model. Here $\sigma_{1}, \ldots$, $\sigma_{21}$ represent neurons, where $\sigma_{i}(i \neq 4,8,12,17,21)$ are proposition neurons, and $\sigma_{4}, \sigma_{8}, \sigma_{12}, \sigma_{17}$ and $\sigma_{21}$ are rule neurons. In addition, $\omega_{1}, \ldots, \omega_{16}$ are output weights, $R_{1}, \ldots, R_{5}$ are fuzzy production rules representing the relationships between the protective device information and line faults, and $c_{1}, \ldots, c_{5}$ are the rule's certainty factors. Next, $L_{S(R) m}, L_{S(R) p}$ and $L_{S(R) s}$ are the main, primary backup and secondary backup protective relays at the sending (or receiving) end, respectively; $C B s$ are the circuit breakers for the protective relays; $L_{S(R) 1}, L_{S(R) 2}$ and $L_{S(R) 3}$ are the main, primary backup and secondary backup protective protections at the sending (or receiving) end, respectively; and $L_{S}$ and $L_{R}$ are protections at the sending and receiving end, respectively. Note that, here, "protections" represent the protective relays and their corresponding circuit breakers in our models for transmission lines, buses and transformers.

(2) Buses

For the bus fault diagnosis model, we create submodels for each exit. Given the way their protective relays operate, our model should include $f$ submodels (one for each exit), and an integrated model.

Fig. 6 illustrates the bus fault diagnosis model. Here, $\sigma_{1}, \ldots, \sigma_{15}$ are neurons, where $\sigma_{i}(i \neq 8,9,10,15)$ are proposition neurons, and $\sigma_{8}, \sigma_{9}, \sigma_{10}$ and $\sigma_{15}$ are rule neurons. In addition, $\omega_{1}, \ldots, \omega_{13}$ are output weights, $R_{1}, \ldots, R_{4}$ are fuzzy production rules representing the relationships between the protective device information and bus faults, and $c_{1}, \ldots, c_{4}$ are the rule's certainty factors. Next, $B_{m}$ and $B_{s 1(2,3)}$ are the main and backup protective relays for the exit 1 (or 2,3 ), $C B s$ represent the circuit breakers for the protective relays, and $B_{1}(2,3)$ is the protection system, including main and backup protections for the exit 1 (or 2, 3). The model shown in Fig. 6 includes three submodels (i.e., $f=3$ ), because we have assumed that the bus has three exits. Note that, in practice, the number of submodels should match the actual number of exits.

(3) Transformers

Given the way transformers' protective relays operate, our fault diagnosis model for a transformer should include main, primary backup and secondary backup protection submodels, and an integrated submodel. In addition, the secondary backup submodel should include separate submodels for both the sending and receiving ends.

Fig. 7 illustrates the transformer fault diagnosis model. Here, $\sigma_{1}, \ldots$, $\sigma_{21}$ represent neurons, where $\sigma_{i}(i \neq 4,8,12,16,21)$ are proposition neurons, and $\sigma_{4}, \sigma_{8}, \sigma_{12}, \sigma_{16}$ and $\sigma_{21}$ are rule neurons. In addition, $\omega_{1}, \ldots, \omega_{16}$ are output weights, $R_{1}, \ldots, R_{5}$ are fuzzy production rules representing the relationships between the protective device information and transformer faults, and $c_{1}, \ldots, c_{5}$ represent the rule's certainty factors. Next, $T_{m}, T_{p}$ and $T_{S(R) s}$ are the main, primary backup, and secondary backup relays at the sending (or receiving) end, respectively, $C B S$ represent the circuit breakers for these relays, and $T_{1}, T_{2}$ and $T_{3(4)}$ are the main, primary backup, and secondary backup protection at the sending (or receiving) end, respectively. 


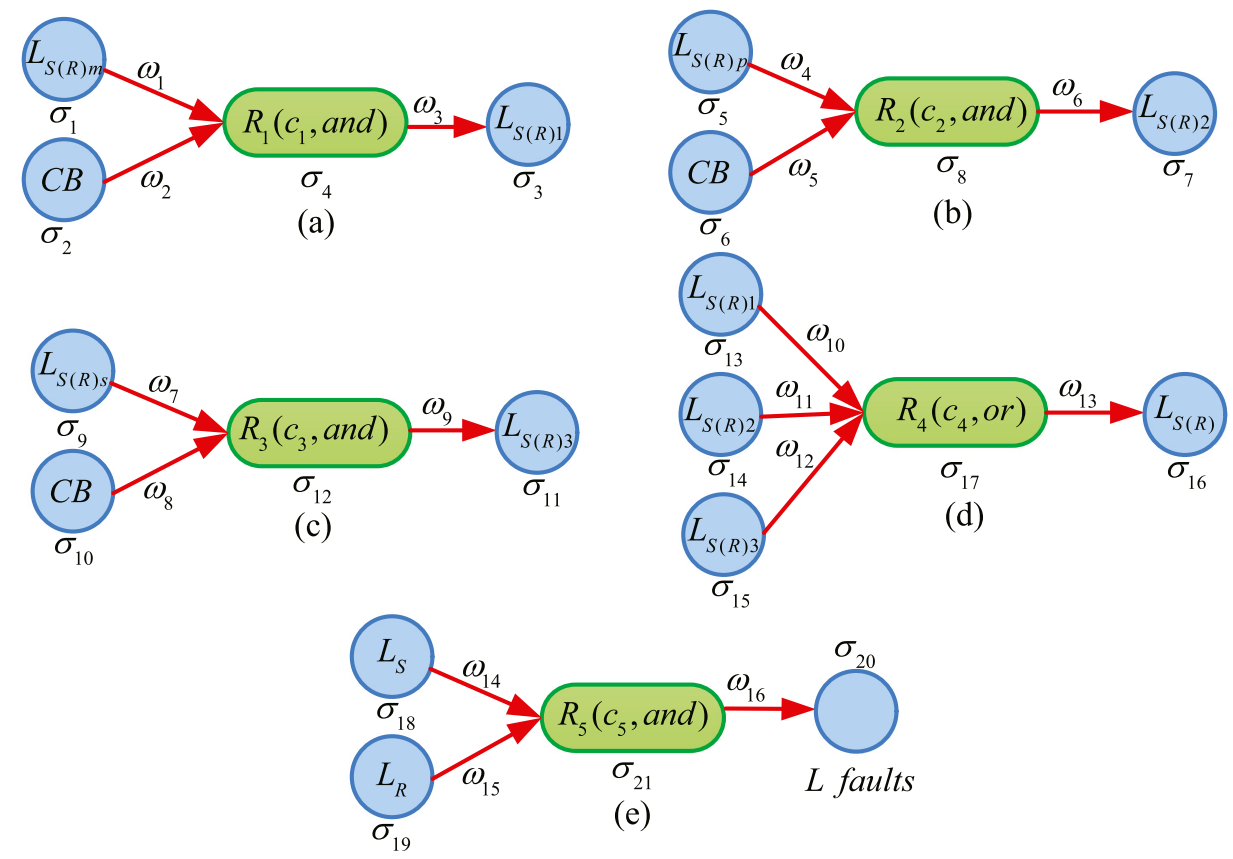

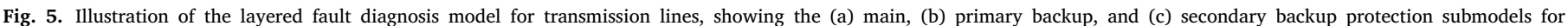
sending (receiving) ends, as well as (d) sending (receiving) end submodels and (e) integrated model combining both ends.

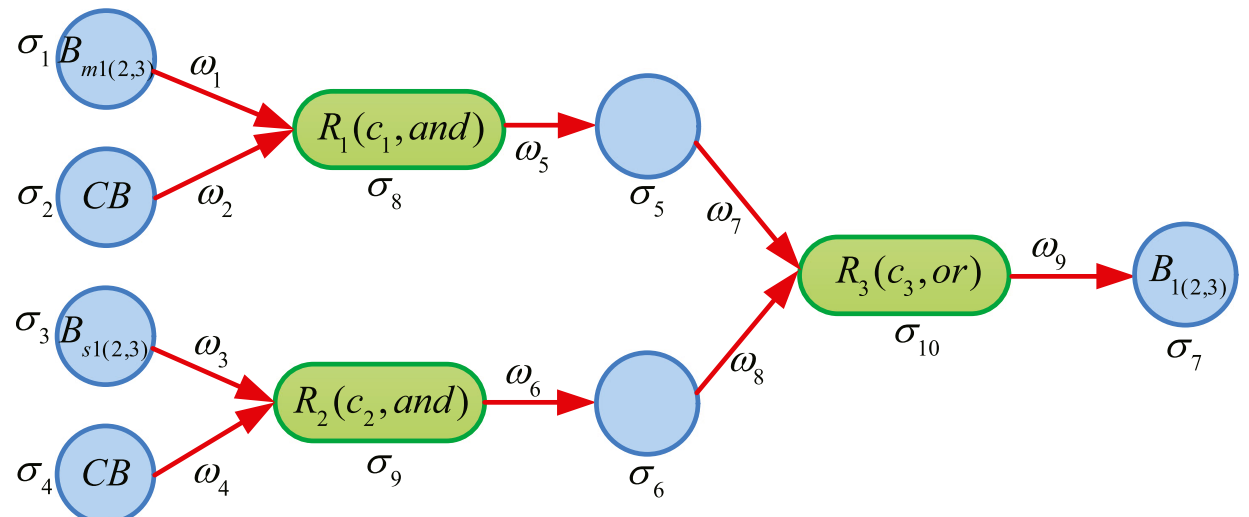

(a)

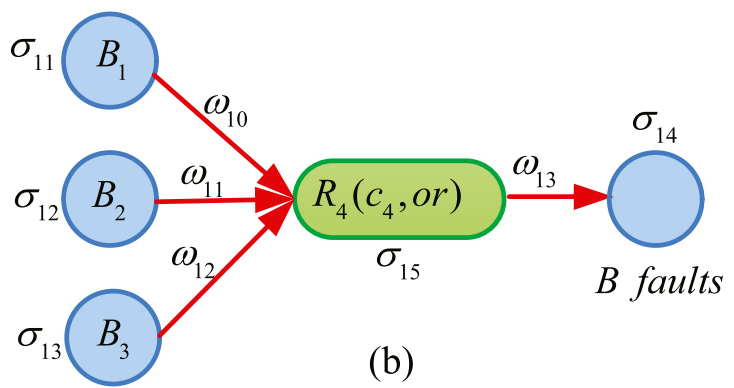

Fig. 6. Illustration of the layered fault diagnosis model for buses. (a) Submodels for the three exits, (b) integrated model.

\subsubsection{Adaptive adjustment rules}

From Section 3.2.1, we know that our layered models all consist of several submodels. Thus, when the power system's topology changes, we do not need to build a complete new diagnosis model, but just modify the submodels associated with the changes. When doing this, transmission lines, buses and transformers have different patterns, which are described below.

Our fault diagnosis models for transmission lines and transformers include nine and six submodels, respectively. Given the way their protective devices operate and the way the models are built, when the power system topology changes, all we need to do in either case is to add or remove the corresponding branches to/from the sending (receiving) end secondary backup protection submodels, not rebuild the whole model. This shows that these layered models are remarkably adaptable to topological changes.

The bus model includes $f+1$ submodels, one for each of the $f$ exits and one integrated submodel. The exit submodels only involve the protective devices associated with the relevant exit. Thus, there are 

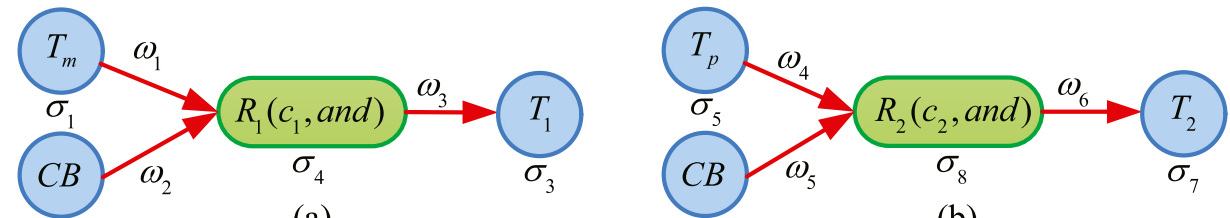

(a)

(b)
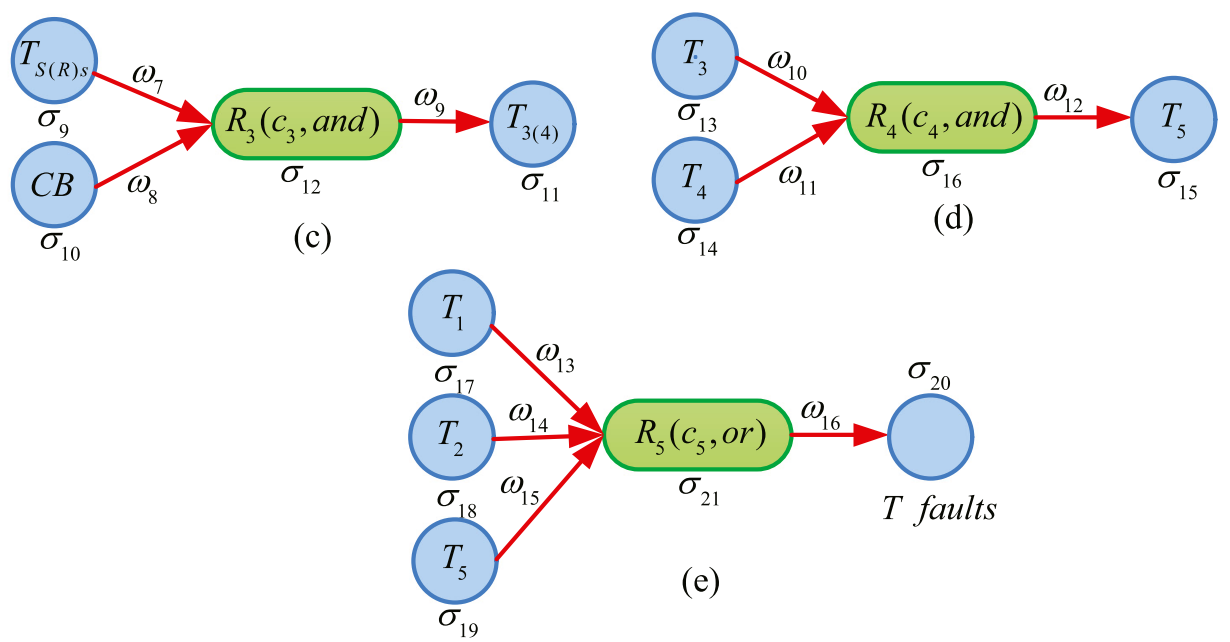

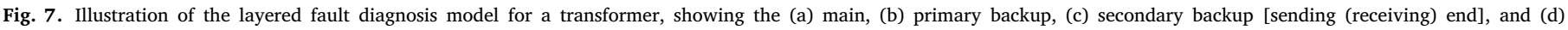
secondary backup (integrated) protection submodels, and the (e) integrated submodel.

different cases to consider in the event of power system topological changes. If the change affects the secondary backup protection for a certain exit, then we need to add (or remove) the corresponding branches to (or from) the corresponding secondary backup protection submodel. If, on the other hand, the exits change, then we just need to add or remove submodels corresponding to the exits. Again, we do not need to rebuild the whole diagnosis model, showing that the layered model for buses is also highly adaptable to topological changes.

\subsubsection{Parameter values}

We use the protective device information obtained from the SCADA system as the inputs of layered fault diagnosis models, but these data is usually incomplete and uncertain because of malfunctions, device failures or the information corruption. Thus, to make the input data more reliable, we need to set a probability value for each protective device, representing our confidence in its reliability. Table 1 in the Appendix shows the protective device confidence levels, both when they are operational and when they are not, where $L, B$ and $T$ represent transmission lines, buses and transformers, respectively. The data in Table 1 is obtained according to the historical statistics of operating statuses of the corresponding protective devices.

Initially, each input neuron only contains one spike, which is a real number in $[0,1]$ ) equaling to the confidence level of its associated protective device. The other neurons do not initially contain any spikes, and hence, their potential values are zero.

Each rule neuron has a truth value representing the associated fuzzy production rule's certainty factor. Usually, the main protective devices are more reliable than the primary backups, which are in turn more reliable than the secondary backups. Thus, on the basis of statistics and expert knowledge, we set the truth values of neurons associated with the main, primary backup and secondary backup protective devices to $0.975,0.95$ and 0.9 , respectively. Note that the truth values of the or rule neurons are set according to their highest protection level. For example, in Fig. 5, the truth values of $\sigma_{4}, \sigma_{8}, \sigma_{12}, \sigma_{17}$ and $\sigma_{21}$ are 0.975, $0.95,0.9,0.975$ and 0.975 , respectively.

For fuzzy production rules, different antecedent propositions have different effects on the consequent propositions, so different proposition neurons (which correspond to antecedent propositions) have different effects on the diagnosis results. We therefore need to set different output weights for each synapse. Given the way protective devices operate, as discussed in Ref. Wang et al. (2015e), we know that when a piece of equipment fails, protective relays trip the corresponding CBs and isolate the fault, indicating that both the protective relays and CBs are very important to handling faults. We therefore set the output weights of proposition neurons corresponding to protective relays to 0.98, those corresponding to circuit breakers to 0.95 , and all other output weights to 1 . For example, the weights $\omega_{1}, \ldots, \omega_{16}$ in Fig. 5 are $0.98,0.95,1,0.98,0.95,1,0.98,0.95,1,1,1,1,1,1,1$ and 1 , respectively.

The firing threshold of each neuron should be smaller than the minimum pulse value it receives during the reasoning process. On the basis of Table 1 in the Appendix and the expert knowledge, to guarantee logical fault knowledge reasoning, we set all the firing thresholds to 0.1. It is worth noting that the above truth values of rule neurons, and the output weights and firing thresholds of neurons are set according to expertise.

\subsection{Processing temporal order information}

Here, we first discuss the temporal order characteristics of SCADA system alarm messages and then present a Temporal Order Information Processing Method based on Cause-Effect Networks (TOIPM-CEN). Finally, a simple power network is used to discuss the TOIPM-CEN in detail.

\subsubsection{Temporal relationships among alarm messages}

When faults occur in power systems, many SCADA system alarm messages pour into the control center in a short period of time. These messages give both the event and temporal relationships between the protective relays and CBs. The relationships between the protective relay and $\mathrm{CB}$ tripping events are usually used to diagnose power system faults, whereas the temporal relationships (i.e., the fact that the corresponding protective relays and CBs should trip within a specific time window after the failure) are important for analyzing the causes of complex faults, fault evolution and protective device behavior. Thus, 


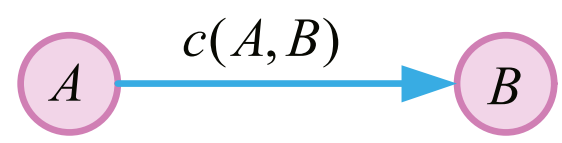

Fig. 8. Simple CEN for the conditional implication rule $R\{c(A, B)\}: A \rightarrow B$.

we could potentially improve diagnosis accuracy by using the temporal relationships between the alarm messages together with the event relationships in the reasoning process. With that in mind, we now consider the temporal relationships between the alarm messages.

When the protective devices operate is determined by both the time delays set for them and the time it takes the protective relays to trip. Specifically, if a fault occurs at time $\tau_{0}$, then the trip time $\tau_{a} \in\left[\tau_{0}+\tau+\tau_{p}^{\min }, \tau_{0}+\tau+\tau_{p}^{\max }\right]$, where $\tau$ is the time delay set for the protective device, $\tau_{p} \in\left[\tau_{p}^{p i n}, \tau_{p}^{\max }\right]$ is the time taken for the relays to trip, and $\tau_{p}^{\min }$ and $\tau_{p}^{\max }$ are the upper bound and lower bound of $\tau_{p}$, respectively. The time delays for the main, primary backup and secondary backup protective systems relative to the failure time are different, at $\tau_{p}$ are $[10,40] \mathrm{ms},[310,340] \mathrm{ms}$ and $[510,450] \mathrm{ms}$, respectively.

Then, at a signal from a protective relay, the corresponding CBs trip. Thus, the time at which they trip is chiefly determined by the time they take to respond to this signal. If a protective relay trips at time $\tau_{a}$ and then the CBs trip after a delay of $\tau_{c}\left(\tau_{c} \in\left[\tau_{c}^{\min }, \tau_{c}^{\max }\right]\right)$, then this should occur in the window $\left[\tau_{a}+\tau_{c}^{\text {min }}, \tau_{a}+\tau_{c}^{\max }\right]$, where $\tau_{c}^{\text {min }}$ and $\tau_{c}^{\text {max }}$ represent the upper bound and lower bound of $\tau_{c}$, respectively. The delay ranges for the CBs corresponding to the main, primary backup and secondary backup relays are all the same, at $[20,40] \mathrm{ms}$.

\subsubsection{TOIPM-CEN}

TOIPM-CENs process temporal order information by using CENs and conditional implication rules in an innovative way to represent the logical relationships between a faulty equipment and the corresponding protective devices (including protective relays and $\mathrm{CBs}$ ), and to depict the causal relationships between nodes. They thus correct the SCADA system alarm messages using both the temporal order information and their conditional implication rules to obtain the information correction parameter matrix $T$ of alarm messages. Before discussing TOIPM-CENs in detail, we must first introduce some of the concepts involved, including event nodes, time constraint relationships between event nodes, and conditional implication rules.

TOIPM-CENs include five types of event nodes, which indicate equipment faults, protective relay activates, circuit breaker trips, protective relay fails to activate, and circuit breaker fails to trip, respectively. These are formally represented as follows: $C_{\text {fault }}(e, \tau)$ represents component $e$ failing at time $\tau$; $C_{\text {operate }}(p, \tau)$ indicates protective relay $p$ activating at time $\tau ; C_{\text {trip }}(c, \tau)$ represents circuit breaker $c$ tripping at time $\tau$; $C_{\text {reject }}(p)$ indicates protective relay $p$ failing to trip at time $\tau$; and $C_{r e j e c t}(c)$ represents circuit breaker $c$ failing to trip at time $\tau$.

TOIPM-CENs represent time constraint relationships between event nodes formally by $\phi^{-}\left(\tau_{1}, \tau_{2}, \tau_{a}, \tau_{b}\right)$ or $\phi^{+}\left(\tau_{1}, \tau_{2}, \tau_{a}, \tau_{b}\right)$, where $\tau_{a} \leq \tau_{2}-$ $\tau_{1} \leq \tau_{b}$. Here, $\tau_{1}$ and $\tau_{2}$ indicate when even nodes 1 and 2 occur, respectively, whereas $\tau_{a}$ and $\tau_{b}$ represent the upper and lower bounds, respectively, on the delay between the two events. In addition, $\phi^{-}$ means that event 1 occurred before event 2 , whereas $\phi^{+}$means that event 2 occurred first. Conditional implication rules are represented by $R\{c(A, B)\}: A \rightarrow B$, where $A$ and $B$ are cause and result event nodes, respectively, and $c(A, B)$ represents a constraint condition between $A$ and $B$. This rule can be described by the simple CEN shown in Fig. 8 .

To illustrate how a TOIPM-CEN corrects alarm messages, we now consider the simple power system, as shown in Fig. 9, where $L_{1 S m}$ and $L_{1 R m}$ are the sending end and receiving end main protectors of line $L$, respectively, $L_{1 S p}$ and $L_{1 R p}$ are corresponding backup protectors, and $C B_{1}$ and $C B_{2}$ are the associated circuit breakers. The TOIPM-CEN is constructed as follows.

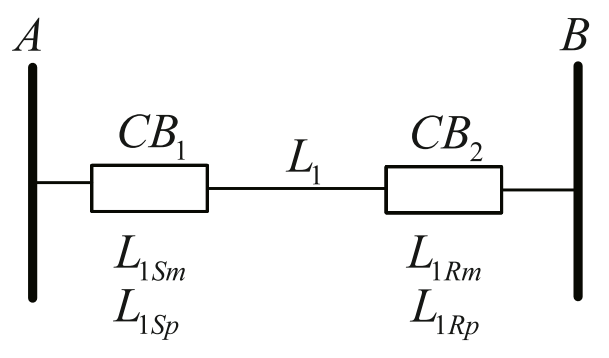

Fig. 9. Simple example power system.

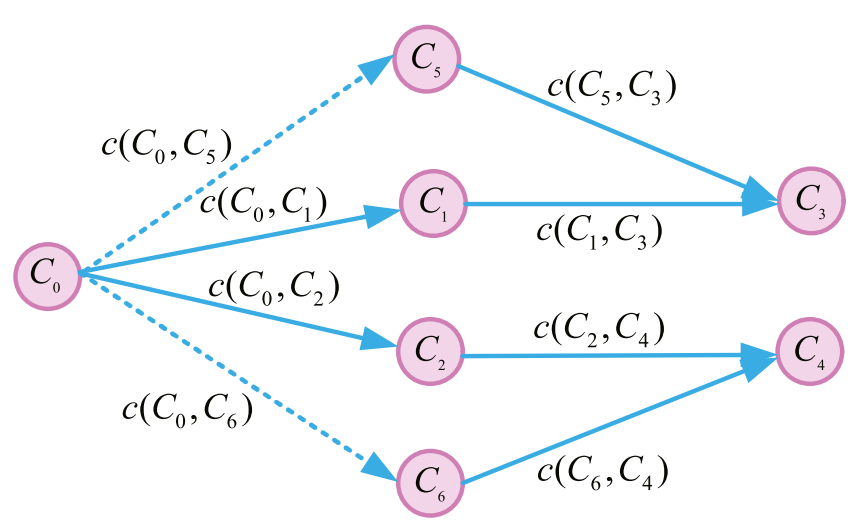

Fig. 10. CEN for the simple power system shown in Fig. 9.

Firstly, we build the CEN shown in Fig. 10 to model the logical relationships between faulty equipment and alarm messages, where $C_{0}, \ldots, C_{6}$ represent a sequence of seven event nodes, namely, $L_{1}$ develops a fault, $L_{1 S m}$ activates, $L_{1 R m}$ activates, $C B_{1}$ trips, $C B_{2}$ trips, $L_{1 S P}$ activates and $L_{1 R P}$ activates, respectively. A solid arrow between $C_{i}$ and $C_{j}$ means that there is only a time constraint between the two nodes, whereas a dotted arrow indicates that there are also other types of relationships between the two nodes. Here, $\tau_{0}, \ldots, \tau_{6}$ represent times that $C_{0}, \ldots, C_{6}$ occur, respectively, and the $c\left(C_{i}, C_{j}\right)(0 \leq i, j \leq$ $6, i \neq j$ ) represents the constraint conditions between events $C_{i}$ and $C_{j}$. The formal representations of the seven event nodes $C_{0}, \ldots, C_{6}$ are shown in Table 2 in the Appendix, and the constraint relationships corresponding to the conditions $c\left(C_{i}, C_{j}\right)(0 \leq i, j \leq 6, i \neq j)$ are given in Table 3, where $\varphi\left(C_{i}, C_{j}\right)=C_{i} \cap \bar{C}_{j}(i=0, j=1,2)$ indicates that event $C_{i}$ occurred, but $C_{j}$ did not. For instance, $\varphi\left(C_{0}, C_{1}\right)$ means that line $L_{1}$ developed a fault but protective relay $L_{1 S m}$ failed to trip.

On the basis of the temporal relationships presented in Section 3.3.1 for alarm messages and ideas discussed in this section, we can derive the conditional implication rule set for the CEN shown in Fig. 10, which is shown in Table 4 in the Appendix. The alarm messages can then be corrected using these implication rules obtained and the temporal relationships. Imagine, for example, that we received the alarm messages, i.e., $C B_{1}(76 \mathrm{~ms})$ and $L_{1 S m}(45 \mathrm{~ms})$, from the SCADA system, indicating that the circuit breaker $C B_{1}$ tripped at $\tau_{3}=76 \mathrm{~ms}$ and protective relay $L_{1 S m}$ activated at $\tau_{1}=45 \mathrm{~ms}$. Then, according to the temporal relationships, the constraints shown in Table 3 and Rule 5 in Table 4, we find that $\tau_{3}-\tau_{1}=76-45=31 \mathrm{~ms}$, meaning that $20 \mathrm{~ms} \leq \tau_{3}-\tau_{1} \leq 40 \mathrm{~ms}$. Thus, $C B_{1}$ tripped correctly, so we set its information correction parameter to 1 .

\subsection{FD-WCFRSNPS}

Now, we summarize our rWCFRSNPS-based fault diagnosis method (FD-WCFRSNPS), which involves the following main steps.

Step 1: Read all fault alarm messages about protective devices from the SCADA system; 


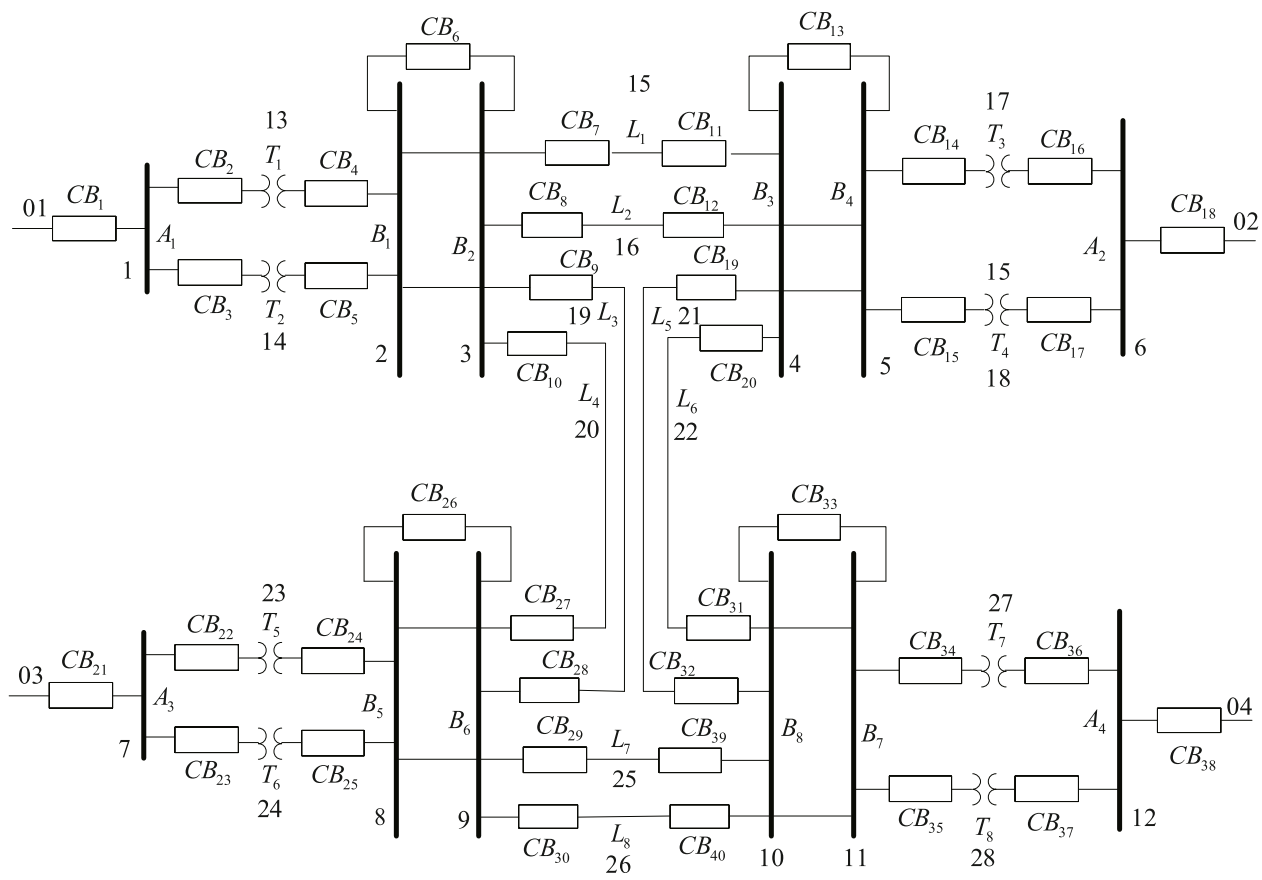

Fig. 11. Overview of a local subsystem of a $220 \mathrm{kV}$ power system.

Step 2: Search for areas with faults. We use a network topologybased analysis method (Wang et al., 2015e; Prais and Bose, 1988) to reduce the number of areas to consider, reducing the computational workload;

Step 3: If there is only one piece of equipment in the areas found in Step 2, then it must be faulty and the algorithm terminates. Otherwise, all equipment in these areas may potentially be faulty, so we create a layered fault diagnosis model based on rWCFRSNPSs for each piece of potentially faulty equipment, as described in Section 3.2.1;

Step 4: Correct and update alarm messages using their temporal relationships and the TOIPM-CEN proposed in Section 3.3.2. Then, obtain the information correction parameter matrix $T$ for each model;

Step 5: Reason about fault, using the MBRA proposed in Section 2.2 to acquire the fault confidence level $\theta$ for each potentially faulty piece of equipment;

Step 6: Find the faulty equipment. A given piece of equipment is faulty if its confidence level $\theta \geq 0.5$; otherwise, it is not.

\section{Case study}

In this section, we test the effectiveness of the FD-WCFRSNPS by considering a local subsystem of a $220 \mathrm{kV}$ power system, previously discussed in Refs. Wen and Han (1995) and Sun et al. (2004), as illustrated in Fig. 11. The system includes 28 pieces of equipment, 84 protective relays and 40 circuit breakers.

The 28 pieces of equipment $\left(S_{1} \sim S_{28}\right)$ are labeled as $A_{1}, \ldots, A_{4}, T_{1}$, $\ldots, T_{8}, B_{1}, \ldots, B_{8}$ and $L_{1}, \ldots, L_{8}$. The 84 protective relays consist of 36 main relays $\left(r_{1} \sim r_{36}\right)$, labeled as $A_{1 m}, \ldots, A_{4 m}, T_{1 m}, \ldots, T_{8 m}, B_{1 m}$, $\ldots, B_{8 m}, L_{1 S m}, \ldots, L_{8 S m}$ and $L_{1 R m}, \ldots, L_{8 R m}$, and 48 backup relays $\left(r_{37} \sim r_{84}\right)$, labeled as $T_{1 p}, \ldots, T_{8 P}, T_{1 s}, \ldots, T_{8 s}, L_{1 S p}, \ldots, L_{8 S p}, L_{1 R p}, \ldots$, $L_{8 R p}, L_{1 S s}, \ldots, L_{8 S s}$ and $L_{1 R s}, \ldots, L_{8 R s}$. Here, $A, B, T$ and $L$ represent single buses, double buses, transformers and lines, respectively; $S$ and $R$ represent the line's sending and receiving ends, respectively; and $m, p$ and $s$ denote the main, primary backup and second backup protective systems, respectively. The 40 circuit breakers $\left(C_{1} \sim C_{40}\right)$ are labeled as $C B_{1}, \ldots, C B_{40}$. The protective devices operate as described in Wang et al. (2015e) and Wen and Han (1995).

Using this system, we consider the five cases shown in Table 5 in the Appendix as examples. In addition, we compare diagnosis results of the FD-WCFRSNPS with those of a Analytic Model (AM) (Zhang et al., 2016), a Fuzzy Petri Net (FPN) (Sun et al., 2004), the rFRSNPS (Peng et al., 2013) and the ivFRSNPS (Wang et al., 2019a) as shown in Table 6 in the Appendix. Table 6 shows that Cases 1 and 2 do not contain uncertain and incomplete alarm messages. So, all the five methods produce the right diagnosis results. In Case 3, the $C B_{6}$ and $C B_{10}$ refused to work. Since the five methods have the uncertainty handling ability, they all find the right faulty equipment. In Case 4, the $C B_{6}$ refused to work and the failure protection $C B_{6 f}$ started to protect the power system. Besides, the time stamp information of the $C B_{9}$ was false. In Case 5, the $L_{2 S m}$ was lost and the $C B_{10}$ was a wrong action. So, we can see that the Cases 4-5 are multiple faults with complex uncertainty in the fault information. Therefore, for Cases 4-5, only the proposed FD-WCFRSNPS and the AM can diagnose the right faults because they correct the wrong fault alarm messages before reasoning or optimization to reduce the uncertain degree of the fault information, which improves their fault tolerance.

To illustrate the steps taken by the FD-WCFRSNPS for this system, we now work through Case 3 as an example.

Case 3: Fault alarm messages: $B_{1 m}, L_{2 R s}$ and $L_{4 R s}$ activated; $C B_{4}$, $C B_{5}, C B_{7}, C B_{9}, C B_{12}$ and $C B_{27}$ tripped. The fault diagnosis algorithm of the FD-WCFRSNPS proceeds as follows.

Step 1: Read above fault alarm messages from the SCADA system.

Step 2: Search for outage areas. Use a network topology-based analysis method to find the set of potentially faulty equipment: $\left\{B_{1}, B_{2}, B_{3}\right.$, $\left.B_{4}\right\}$.

Step 3: Because Step 2 found more than one piece of potentially faulty equipment, build a layered fault diagnosis model for each piece of equipment in the set. Here, taking bus $B_{1}$ as an example, the corresponding model is shown in Fig. 12, where Fig. 12(e) and (f) give the submodels built for the bus tie switch $\mathrm{CB}_{6}$.

Step 4: Process the alarm messages and then obtain the matrix $T$ for each model. Here, we show how the proposed TOIPM-CEN uses temporal order information to process the alarm messages, taking bus $B_{1}$ as an example.

(1) Describe the logical relationships between $B_{1}$ failing and the protective devices sending out alarm messages using the CEN shown in Fig. 13. Here, $C_{0}, \ldots, C_{17}$ represent 18 event nodes, whose formal representations are shown in Table 7 in the Appendix, where $B_{F}$ represents a protection failure. 

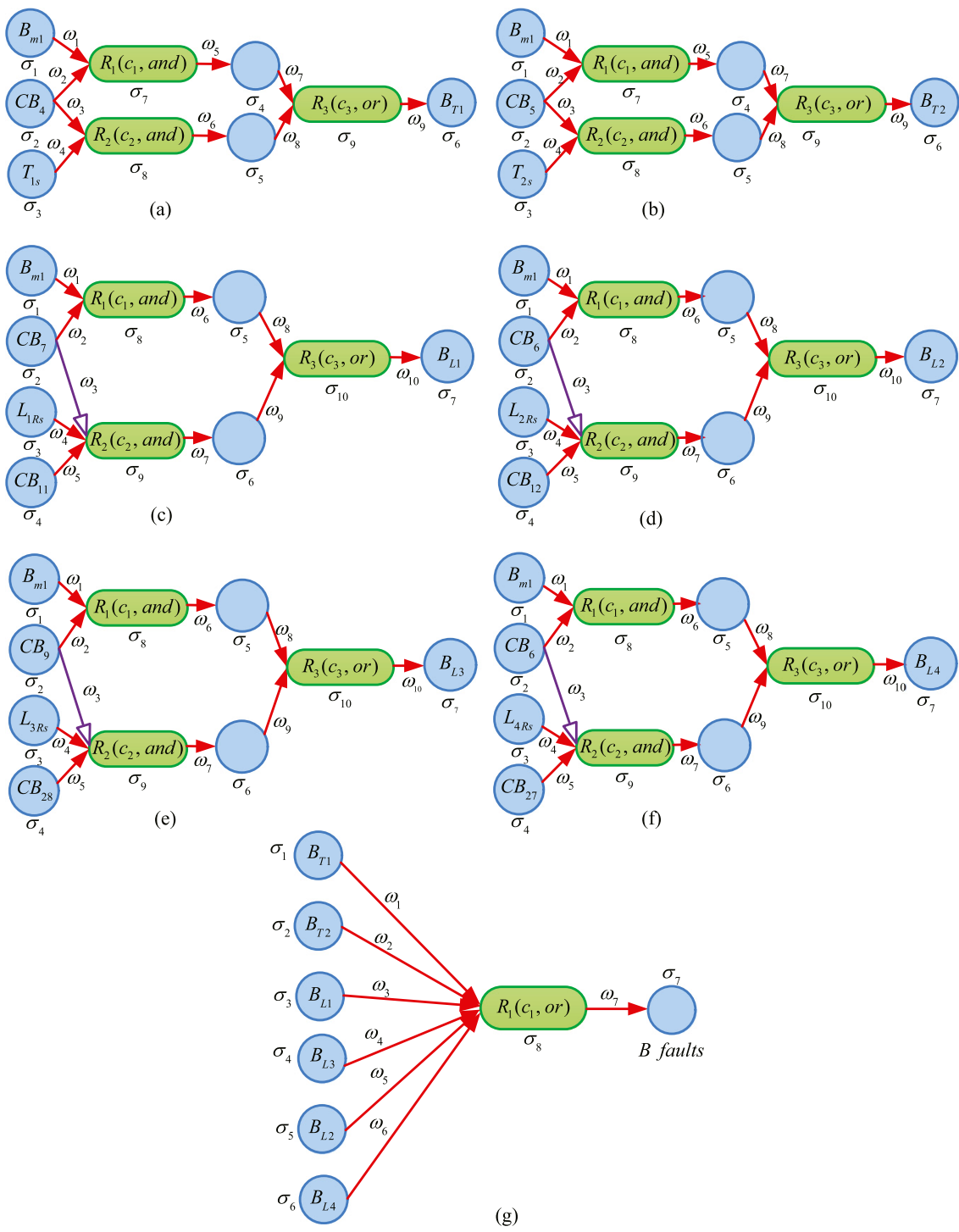

Fig. 12. The layered fault diagnosis model for bus $B_{1}$, showing the submodels for exits on (a) $T_{1}$, (b) $T_{2}$, (c) $L_{1}$, (d) $L_{2}$, (e) $L_{3}$ and (f) $L_{4}$; and the (g) integrated model.

(2) Derive the conditional implication rule set for the CEN shown in Fig. 13, as shown in Table 8 in the Appendix.

(3) On the basis of the temporal relationships and the rule set shown in Table 8, we find that $C B_{6}$ failed to trip, but the rest of the protective devices worked correctly.

(4) Obtain the information correction parameter matrix $T$ for each model built in Step 3. Here, we take the model for $B_{1}$ (shown in Fig. 12) as an example. This model includes seven submodels, whose information correction parameter matrices are as follows: $\mathbf{T}_{1}=\operatorname{diag}(1,1,1,0,0$, $0) ; \mathbf{T}_{2}=\operatorname{diag}(1,1,1,0,0,0) ; \mathbf{T}_{3}=\operatorname{diag}(1,1,1,1,0,0,0) ; \mathbf{T}_{4}=\operatorname{diag}(1,1,1,1$, $0,0,0) ; \mathbf{T}_{5}=\operatorname{diag}(1,1,1,1,0,0,0) ; \mathbf{T}_{6}=\operatorname{diag}(1,1,1,1,0,0,0) ;$ and $\mathbf{T}_{7}=$ $\operatorname{diag}(1,1,1,1,1,1,0)$.

Step 5: Apply the MBRA to each fault diagnosis model to calculate the fault confidence level for each piece of potentially faulty equipment. Again, we consider bus $B_{1}$ as an example and apply the MBRA to each of the submodels in Fig. 12(a)-(f) in parallel. We have to consider the integrated model (Fig. 12(g)) after the other six submodels because it takes their outputs as inputs. Next, we describe how the MBRA acquires the fault confidence levels for each piece of equipment, using the submodel on exit $T_{1}$. The parameter matrices for the submodel are as follows:

$\mathbf{W}_{p}=\left[\begin{array}{llllll}0 & 0 & 0 & 1 & 0 & 0 \\ 0 & 0 & 0 & 0 & 1 & 0 \\ 0 & 0 & 0 & 0 & 0 & 1\end{array}\right], \quad \mathbf{W}_{r 1}=[\mathbf{O}]_{6 \times 3}$,

$$
\begin{aligned}
& \mathbf{W}_{r 2}=\left[\begin{array}{cccccc}
0.98 & 0.95 & 0 & 0 & 0 & 0 \\
0 & 0.95 & 0.98 & 0 & 0 & 0 \\
0 & 0 & 0 & 0 & 0 & 0
\end{array}\right]^{T}, \\
& \mathbf{W}_{r 3}=\left[\begin{array}{llllll}
0 & 0 & 0 & 0 & 0 & 0 \\
0 & 0 & 0 & 0 & 0 & 0 \\
0 & 0 & 0 & 1 & 1 & 0
\end{array}\right]^{T}, \mathbf{D}_{1}=\left[\begin{array}{llllll}
1 & 1 & 0 & 0 & 0 & 0 \\
0 & 1 & 1 & 0 & 0 & 0 \\
0 & 0 & 0 & 1 & 1 & 1
\end{array}\right]^{T}, \\
& \mathbf{D}_{2}=\left[\begin{array}{llllll}
0 & 0 & 0 & 1 & 0 & 0 \\
0 & 0 & 0 & 0 & 1 & 0 \\
0 & 0 & 0 & 0 & 0 & 1
\end{array}\right], \\
& \mathbf{C}=\operatorname{diag}(0.975,0.9,0.975), \mathbf{T}_{1}=\operatorname{diag}(1,1,1,0,0,0), \\
& \boldsymbol{\lambda}_{p}=(0.1,0.1,0.1,0.1,0.1,0.1)^{T}, \\
& \mathbf{\theta}_{p}^{0}=(0.8504,0.9833,0.7,0,0,0)^{T}, \mathbf{a}_{p}^{0}=(1,1,1,0,0,0)^{T} .
\end{aligned}
$$

When $g=0$, we obtain the reasoning results: $\theta_{r}^{1}=(0.8334,0.686,0)^{T}$ and $\theta_{p}^{1}=(0,0,0,0.8127,0.6174,0)^{T}$. Then, when $g=1$, we obtain $\theta_{r}^{2}$ $=(0,0,0.8127)^{T}$ and $\theta_{p}^{2}=(0,0,0,0,0,0.7924)^{T}$, and for $g=2$, we obtain $\theta_{r}^{3}=(0,0,0)^{T}$ and $\theta_{p}^{3}=(0,0,0,0,0,0)^{T}$. At this point $(g=2)$ the MBRA's termination condition is satisfied and reasoning ends. Output neuron $\sigma_{6}$ in Fig. 12(a) then yields its potential value, namely, 0.7924. This means that the faulty confidence level of a fault in $B_{1}$ on exit $T_{1}$ is 0.7924 .

Similarly, we obtain the fault confidence levels of a fault in $B_{1}$ on exits $T_{2}, L_{1}, L_{2}, L_{3}$ and $L_{4}$, namely, 0.7924, 0.7924, $0.6252,0.7924$ 
Table 1

Operation and no-operation confidence levels of the protective devices.

\begin{tabular}{|c|c|c|c|c|c|c|c|c|c|c|c|c|}
\hline \multirow[t]{3}{*}{ Equipments } & \multicolumn{6}{|c|}{ Protective devices (operation) } & \multicolumn{6}{|c|}{ Protective devices (no-operation) } \\
\hline & \multicolumn{2}{|l|}{ Main } & \multicolumn{2}{|c|}{ First backup } & \multicolumn{2}{|c|}{ Second backup } & \multicolumn{2}{|l|}{ Main } & \multicolumn{2}{|c|}{ First backup } & \multicolumn{2}{|c|}{ Second backup } \\
\hline & Relays & CBs & Relays & CBs & Relays & CBs & Relays & $\overline{\mathrm{CBs}}$ & Relays & CBs & Relays & CBs \\
\hline$L$ & 0.9913 & 0.9833 & 0.8 & 0.85 & 0.7 & 0.75 & 0.2 & 0.2 & 0.2 & 0.2 & 0.2 & 0.2 \\
\hline$B$ & 0.8564 & 0.9833 & - & - & 0.7 & 0.75 & 0.4 & 0.2 & - & - & 0.4 & 0.2 \\
\hline$T$ & 0.7756 & 0.9833 & 0.75 & 0.8 & 0.7 & 0.75 & 0.4 & 0.2 & 0.4 & 0.2 & 0.4 & 0.2 \\
\hline
\end{tabular}

Table 2

The formal representation of node events $C_{0}$ to $C_{6}$.

\begin{tabular}{llll}
\hline Symbol & Formal representation & Symbol & Formal representation \\
\hline$C_{0}$ & $C_{\text {fault }}\left(L_{1}, \tau_{0}\right)$ & $C_{4}$ & $C_{\text {trip }}\left(C B_{2}, \tau_{4}\right)$ \\
$C_{1}$ & $C_{\text {operate }}\left(L_{1 S m}, \tau_{1}\right)$ & $C_{5}$ & $C_{\text {operate }}\left(L_{1 S p}, \tau_{5}\right)$ \\
$C_{2}$ & $C_{\text {operate }}\left(L_{1 R m}, \tau_{2}\right)$ & $C_{6}$ & $C_{\text {operate }}\left(L_{1 R p}, \tau_{6}\right)$ \\
$C_{3}$ & $C_{\text {trip }}\left(C B_{1}, \tau_{3}\right)$ & - & - \\
\hline
\end{tabular}

Table 3

The implications of the constraint conditions.

\begin{tabular}{|c|c|c|c|}
\hline Symbol & Implication & Symbol & Implication \\
\hline $\mathrm{c}\left(\mathrm{C}_{0}, \mathrm{C}_{1}\right)$ & $\phi^{-}\left(\tau_{0}, \tau_{1}, 10 \mathrm{~ms}, 40 \mathrm{~ms}\right)$ & $\mathrm{c}\left(\mathrm{C}_{1}, \mathrm{C}_{3}\right)$ & $\phi^{-}\left(\tau_{1}, \tau_{3}, 20 \mathrm{~ms}, 40 \mathrm{~ms}\right)$ \\
\hline $\mathrm{c}\left(\mathrm{C}_{0}, \mathrm{C}_{2}\right)$ & $\phi^{-}\left(\tau_{0}, \tau_{2}, 10 \mathrm{~ms}, 40 \mathrm{~ms}\right)$ & $\mathrm{c}\left(\mathrm{C}_{2}, \mathrm{C}_{4}\right)$ & $\phi^{-}\left(\tau_{2}, \tau_{4}, 20 \mathrm{~ms}, 40 \mathrm{~ms}\right)$ \\
\hline $\mathrm{c}\left(\mathrm{C}_{0}, \mathrm{C}_{5}\right)$ & $\phi^{-}\left(\tau_{0}, \tau_{5}, 310 \mathrm{~ms}, 340 \mathrm{~ms}\right), \quad \varphi\left(C_{0}, C_{1}\right)$ & $\mathrm{c}\left(\mathrm{C}_{5}, \mathrm{C}_{3}\right)$ & $\phi^{-}\left(\tau_{5}, \tau_{3}, 20 \mathrm{~ms}, 40 \mathrm{~ms}\right)$ \\
\hline $\mathrm{c}\left(\mathrm{C}_{0}, \mathrm{C}_{6}\right)$ & $\phi^{-}\left(\tau_{0}, \tau_{6}, 310 \mathrm{~ms}, 340 \mathrm{~ms}\right), \quad \varphi\left(C_{0}, C_{2}\right)$ & $\mathrm{c}\left(\mathrm{C}_{6}, \mathrm{C}_{4}\right)$ & $\phi^{-}\left(\tau_{6}, \tau_{4}, 20 \mathrm{~ms}, 40 \mathrm{~ms}\right)$ \\
\hline
\end{tabular}

Table 4

Implication rule set with conditional constraint for the CNE in Fig. 4.

\begin{tabular}{ll}
\hline Number & Rule \\
\hline 1 & $R_{1}\left\{\phi^{-}\left(\tau_{0}, \tau_{1}, 10 \mathrm{~ms}, 40 \mathrm{~ms}\right)\right\}: C_{\text {fault }}\left(L_{1}, \tau_{0}\right) \rightarrow C_{\text {operate }}\left(L_{1 S m}, \tau_{1}\right)$ \\
2 & $R_{2}\left\{\phi^{-}\left(\tau_{0}, \tau_{2}, 10 \mathrm{~ms}, 40 \mathrm{~ms}\right)\right\}: C_{\text {fault }}\left(L_{1}, \tau_{0}\right) \rightarrow C_{\text {operate }}\left(L_{1 R m}, \tau_{2}\right)$ \\
3 & $R_{3}\left\{\phi^{-}\left(\tau_{0}, \tau_{5}, 310 \mathrm{~ms}, 340 \mathrm{~ms}\right), \varphi\left(C_{0}, C_{1}\right)\right\}: C_{\text {fault }}\left(L_{1}, \tau_{0}\right) \rightarrow C_{\text {operate }}\left(L_{1 S p}, \tau_{5}\right)$ \\
4 & $R_{4}\left\{\phi^{-}\left(\tau_{0}, \tau_{6}, 310 \mathrm{~ms}, 340 \mathrm{~ms}\right), \varphi\left(C_{0}, C_{2}\right)\right\}: C_{\text {fault }}\left(L_{1}, \tau_{0}\right) \rightarrow C_{\text {operate }}\left(L_{1 R p}, \tau_{6}\right)$ \\
5 & $R_{5}\left\{\phi^{-}\left(\tau_{1}, \tau_{3}, 20 \mathrm{~ms}, 40 \mathrm{~ms}\right)\right\}: C_{\text {operate }}\left(L_{1 S m}, \tau_{1}\right) \rightarrow C_{\text {trip }}\left(C B_{1}, \tau_{3}\right)$ \\
6 & $R_{6}\left\{\phi^{-}\left(\tau_{2}, \tau_{4}, 20 \mathrm{~ms}, 40 \mathrm{~ms}\right)\right\}: C_{\text {operate }}\left(L_{1 R m}, \tau_{2}\right) \rightarrow C_{\text {trip }}\left(C B_{2}, \tau_{4}\right)$ \\
7 & $R_{7}\left\{\phi^{-}\left(\tau_{5}, \tau_{3}, 20 \mathrm{~ms}, 40 \mathrm{~ms}\right)\right\}: C_{\text {operate }}\left(L_{1 S p}, \tau_{5}\right) \rightarrow C_{\text {trip }}\left(C B_{1}, \tau_{3}\right)$ \\
8 & $R_{8}\left\{\phi^{-}\left(\tau_{6}, \tau_{4}, 20 \mathrm{~ms}, 40 \mathrm{~ms}\right)\right\}: C_{\text {operate }}\left(L_{1 R p}, \tau_{6}\right) \rightarrow C_{\text {trip }}\left(C B_{2}, \tau_{4}\right)$ \\
\hline
\end{tabular}

and 0.6252 , respectively. Finally, we obtain the overall fault confidence level for $B_{1}$ by applying the MBRA to the integrated model in Fig. 12(g), yielding 0.7924 .

Step 6: According to the condition for Step 6 in Section $3.4(\theta>0.5)$, bus $B_{1}$ has developed a fault, because its fault confidence level is 0.7924 (> 0.5)

If we likewise carry out Steps 1-6 for bus $B_{2}$ and lines $L_{2}$ and $L_{4}$, we find that $B_{2}, L_{2}$ and $L_{4}$ did not fail. Thus, for Case 3 , the diagnosis result is that only bus $B_{1}$ failed, with a fault confidence level of 0.7924 .

\section{Conclusion and future work}

Estimating which equipment is faulty is of great importance when attempting to restore power systems after failures. In this paper, we have proposed a graphical fault estimation method for power systems called FD-WCFRSNPS, based on rWCFRSNPSs. This method uses rWCFRSNPSs to represent fuzzy fault alarm information and the MBRA for reasoning, together with a temporal order information processing method called TOIPM-CEN that corrects the alarm information. In addition, we develop a layered approach to building fault diagnosis models based on rWCFRSNPSs that enables them to adapt to changes in power system topology, discussing in detail how the models are built and the adaptive adjustment rules are derived. In addition, we discuss suitable parameter values for transmission lines, buses and transformers.

The FD-WCFRSNPS provides highly accurate diagnoses, thanks to the TOIPM-CEN and layered fault diagnosis models. In addition, the proposed modeling method makes the fault diagnosis process easier to understand, illustrating it with intuitive graphical layered models,

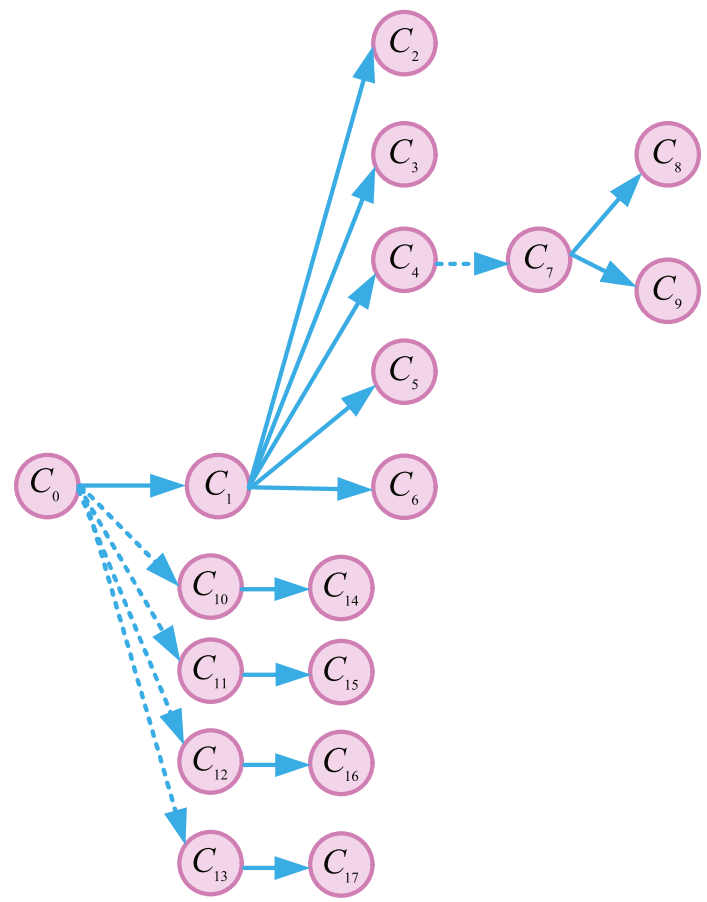

Fig. 13. The CEN representing the logical relationships between $B_{1}$ failing and the corresponding alarm messages.

providing good expressions for the relationships between faults and protective devices, and offering an intelligible and adaptive diagnosis model-building process. Our method avoids the weakness of the empirical values used by trapezoidal fuzzy number (Wang et al., 2015e) and intuitionistic fuzzy number (Peng et al., 2018) approaches. In addition, the FD-WCFRSNPS is suitable for large power systems or power grids with variable topologies, such as those involving distributed generation (DG) or microgrids.

This study has proposed the FD-WCFRSNPS and tested its feasibility and effectiveness for estimating faulty equipment in power systems by working through a detailed implementation. As a case study, we have considered a typical $220 \mathrm{kV}$ power system. We are currently working on applying the FD-WCFRSNPS to large-scale power systems and power grids with DG or microgrids. Until now, SNPS-based fault diagnosis methods have focused on using remote signals, but our future work will focus on using such a method for data-driven fault diagnosis with multisource fault information (Fan and Liao, 2019) and analyzing complex problems in smart grids from the perspective of complex networks (Wen and Deng, 2020, 2019). Besides, the work in this paper is done based on the normal operation of the SCADA system without regard for the information transmission time delay, communication failures and hacker attacks. So, the fault diagnosis of power systems considering extreme cases, such as communication failures, attacks and strong interdependencies between communication networks and physical networks in power systems, is also our important future research directions. 
Table 5

Fault alarm messages of protective devices for cases 1-5

\begin{tabular}{lll}
\hline \multirow{2}{*}{ Cases } & Protective devices & \\
\cline { 2 - 3 } & Protective relays & Circuit breakers \\
\hline 1 & $L_{2 S m}(31 \mathrm{~s}), L_{2 R m}(32 \mathrm{~ms})$ & $C B_{8}(64 \mathrm{~ms}), C B_{12}(66 \mathrm{~ms})$ \\
\hline 2 & $B_{1 m}(20 \mathrm{~ms}), L_{2 S m}(23 \mathrm{~ms}), L_{2 R m}(24 \mathrm{~ms})$ & $C B_{6}(49 \mathrm{~ms}), C B_{4}(50 \mathrm{~ms}), C B_{5}(51 \mathrm{~ms}), C B_{7}(51 \mathrm{~ms})$ \\
\hline 3 & $B_{1 m}(50 \mathrm{~ms}), L_{2 R s}(563 \mathrm{~ms}), C B_{8}(56 \mathrm{~ms}), C B_{12}(57 \mathrm{~ms})$ \\
\hline 4 & $B_{1 m}(20 \mathrm{~ms}), L_{2 S m}(23 \mathrm{~ms}), L_{2 R m}(24 \mathrm{~ms})$ & $C B_{4}(85 \mathrm{~ms}), C B_{5}(87 \mathrm{~ms}), C B_{7}(83 \mathrm{~ms})$, \\
\hline 5 & $B_{1 m}(20 \mathrm{~ms}), L_{2 R m}(24 \mathrm{~ms})$ & $C B_{9}(84 \mathrm{~ms}), C B_{12}(585 \mathrm{~ms}), C B_{27}(600 \mathrm{~ms})$ \\
\hline
\end{tabular}

Table 6

Comparisons between the FDWPSNP and four other methods.

\begin{tabular}{|c|c|c|c|c|c|c|c|}
\hline \multirow[t]{2}{*}{ Cases } & \multirow{2}{*}{$\begin{array}{l}\text { Faulty } \\
\text { equipment }\end{array}$} & \multicolumn{5}{|l|}{ Diagnosis results } & \multirow{2}{*}{$\begin{array}{l}\text { Information } \\
\text { evaluation }\end{array}$} \\
\hline & & FD-WCFRSNPS & $\begin{array}{l}\text { AM (Zhang } \\
\text { et al., 2016) }\end{array}$ & $\begin{array}{l}\text { FPN (Sun et al., } \\
\text { 2004) }\end{array}$ & $\begin{array}{l}\text { rFRSNPS (Peng } \\
\text { et al., 2013) }\end{array}$ & $\begin{array}{l}\text { ivFRSNPS (Wang } \\
\text { et al., 2019a) }\end{array}$ & \\
\hline 1 & $L_{2}$ & $L_{2}$ & $L_{2}$ & $L_{2}$ & $L_{2}$ & $L_{2}$ & correct \\
\hline 2 & $B_{1}, L_{2}$ & $B_{1}, L_{2}$ & $B_{1}, L_{2}$ & $B_{1}, L_{2}$ & $B_{1}, L_{2}$ & $B_{1}, L_{2}$ & correct \\
\hline 3 & $B_{1}$ & $B_{1}$ & $B_{1}$ & $B_{1}$ & $B_{1}$ & $B_{1}$ & $C B_{6}, C B_{10}:$ refused \\
\hline 4 & $B_{1}, L_{2}$ & $B_{1}, L_{2}$ & $B_{1}, L_{2}$ & $L_{2}$ & $L_{2}$ & $L_{2}$ & $C B_{6}:$ refused; $C B_{9}:$ false time \\
\hline 5 & $B_{1}, L_{2}$ & $B_{1}, L_{2}$ & $B_{1}, L_{2}$ & $B_{1}, B_{2}, L_{2}$ & $B_{1}, B_{2}, L_{2}$ & $B_{1}, B_{2}, L_{2}$ & $L_{2 S m}:$ lost $; C B_{10}:$ wrong operation \\
\hline
\end{tabular}

Table 7

Formal representation of event nodes shown in Fig. 13.

\begin{tabular}{llllll}
\hline Symbol & $\begin{array}{l}\text { Formal } \\
\text { representation }\end{array}$ & Symbol & $\begin{array}{l}\text { Formal } \\
\text { representation }\end{array}$ & Symbol & $\begin{array}{l}\text { Formal } \\
\text { representation }\end{array}$ \\
\hline$C_{0}$ & $C_{\text {fault }}\left(B_{1}, \tau_{0}\right)$ & $C_{6}$ & $C_{\text {trip }}\left(C B_{9}, \tau_{6}\right)$ & $C_{12}$ & $C_{\text {operate }}\left(L_{4 R s}, \tau_{12}\right)$ \\
$C_{1}$ & $C_{\text {operate }}\left(B_{1 m}, \tau_{1}\right)$ & $C_{7}$ & $C_{\text {operate }}\left(B_{F}, \tau_{7}\right)$ & $C_{13}$ & $C_{\text {operate }}\left(L_{3 R s}, \tau_{13}\right)$ \\
$C_{2}$ & $C_{\text {trip }}\left(C B_{4}, \tau_{2}\right)$ & $C_{8}$ & $C_{\text {trip }}\left(C B_{8}, \tau_{8}\right)$ & $C_{14}$ & $C_{\text {trip }}\left(C B_{11}, \tau_{14}\right)$ \\
$C_{3}$ & $C_{\text {trip }}\left(C B_{5}, \tau_{3}\right)$ & $C_{9}$ & $C_{\text {trip }}\left(C B_{10}, \tau_{9}\right)$ & $C_{15}$ & $C_{\text {trip }}\left(C B_{12}, \tau_{15}\right)$ \\
$C_{4}$ & $C_{\text {trip }}\left(C B_{6}, \tau_{4}\right)$ & $C_{10}$ & $C_{\text {operate }}\left(L_{1 R s}, \tau_{10}\right)$ & $C_{16}$ & $C_{\text {trip }}\left(C B_{27}, \tau_{16}\right)$ \\
$C_{5}$ & $C_{\text {trip }}\left(C B_{7}, \tau_{5}\right)$ & $C_{11}$ & $C_{\text {operate }}\left(L_{2 R s}, \tau_{11}\right)$ & $C_{17}$ & $C_{\text {trip }}\left(C B_{28}, \tau_{17}\right)$ \\
\hline
\end{tabular}

\section{Table 8}

Conditional implication rule set for the CEN shown in Fig. 13.

\section{Number Rule}

\begin{tabular}{|c|c|}
\hline 1 & $R_{1}\left\{\phi^{-}\left(\tau_{0}, \tau_{1}, 10 \mathrm{~ms}, 40 \mathrm{~ms}\right)\right\}: C_{\text {fault }}\left(B_{1}, \tau_{0}\right) \rightarrow C_{\text {operate }}\left(B_{1 m}, \tau_{1}\right)$ \\
\hline 2 & $R_{2}\left\{\phi^{-}\left(\tau_{1}, \tau_{2}, 20 \mathrm{~ms}, 40 \mathrm{~ms}\right)\right\}: C_{\text {operate }}\left(B_{1 m}, \tau_{1}\right) \rightarrow C_{\text {trip }}\left(C B_{4}, \tau_{2}\right)$ \\
\hline 3 & $R_{3}\left\{\phi^{-}\left(\tau_{1}, \tau_{3}, 20 \mathrm{~ms}, 40 \mathrm{~ms}\right)\right\}: C_{\text {operate }}\left(B_{1 m}, \tau_{1}\right) \rightarrow C_{\text {trip }}\left(C B_{5}, \tau_{3}\right)$ \\
\hline 4 & $R_{4}\left\{\phi^{-}\left(\tau_{1}, \tau_{4}, 20 \mathrm{~ms}, 40 \mathrm{~ms}\right)\right\}: C_{\text {operate }}\left(B_{1 m}, \tau_{1}\right) \rightarrow C_{\text {trip }}\left(C B_{6}, \tau_{4}\right)$ \\
\hline 5 & $R_{5}\left\{\phi^{-}\left(\tau_{1}, \tau_{5}, 20 \mathrm{~ms}, 40 \mathrm{~ms}\right)\right\}: C_{\text {operate }}\left(B_{1 m}, \tau_{1}\right) \rightarrow C_{\text {trip }}\left(C B_{7}, \tau_{5}\right)$ \\
\hline 6 & $R_{6}\left\{\phi^{-}\left(\tau_{1}, \tau_{6}, 20 \mathrm{~ms}, 40 \mathrm{~ms}\right)\right\}: C_{\text {operate }}\left(B_{1 m}, \tau_{1}\right) \rightarrow C_{\text {trip }}\left(C B_{9}, \tau_{6}\right)$ \\
\hline 7 & $R_{7}\left\{\phi^{-}\left(\tau_{0}, \tau_{7}, 310 \mathrm{~ms}, 340 \mathrm{~ms}\right), \varphi\left(C_{1}, C_{4}\right)\right\}: C_{\text {fault }}\left(B_{1}, \tau_{0}\right) \rightarrow C_{\text {operate }}\left(B_{F}, \tau_{7}\right)$ \\
\hline 8 & $R_{8}\left\{\phi^{-}\left(\tau_{7}, \tau_{8}, 20 \mathrm{~ms}, 40 \mathrm{~ms}\right)\right\}: C_{\text {operate }}\left(B_{F}, \tau_{7}\right) \rightarrow C_{\text {trip }}\left(C B_{8}, \tau_{8}\right)$ \\
\hline 9 & $R_{9}\left\{\phi^{-}\left(\tau_{7}, \tau_{9}, 20 \mathrm{~ms}, 40 \mathrm{~ms}\right)\right\}: C_{\text {operate }}\left(B_{F}, \tau_{7}\right) \rightarrow C_{\text {trip }}\left(C B_{10}, \tau_{9}\right)$ \\
\hline 10 & $R_{10}\left\{\phi^{-}\left(\tau_{0}, \tau_{10}, 510 \mathrm{~ms}, 40 \mathrm{~ms}\right), \varphi\left(C_{0}, C_{1}\right)\right\}: C_{\text {fault }}\left(B_{1}, \tau_{0}\right) \rightarrow C_{\text {operate }}\left(L_{1 R s}, \tau_{10}\right)$ \\
\hline 11 & $R_{11}\left\{\phi^{-}\left(\tau_{0}, \tau_{11}, 510 \mathrm{~ms}, 540 \mathrm{~ms}\right), \varphi\left(C_{0}, C_{1}\right)\right\}: C_{\text {fault }}\left(B_{1}, \tau_{0}\right) \rightarrow C_{\text {operate }}\left(L_{2 R s}, \tau_{11}\right)$ \\
\hline 12 & $R_{12}\left\{\phi^{-}\left(\tau_{0}, \tau_{12}, 510 \mathrm{~ms}, 540 \mathrm{~ms}\right), \varphi\left(C_{0}, C_{1}\right)\right\}: C_{\text {fault }}\left(B_{1}, \tau_{0}\right) \rightarrow C_{\text {operate }}\left(L_{4 R s}, \tau_{12}\right)$ \\
\hline 13 & $R_{13}\left\{\phi^{-}\left(\tau_{0}, \tau_{13}, 510 \mathrm{~ms}, 540 \mathrm{~ms}\right), \varphi\left(C_{0}, C_{1}\right)\right\}: C_{\text {fault }}\left(B_{1}, \tau_{0}\right) \rightarrow C_{\text {operate }}\left(L_{3 R s}, \tau_{13}\right)$ \\
\hline 14 & $R_{14}\left\{\phi^{-}\left(\tau_{10}, \tau_{14}, 20 \mathrm{~ms}, 40 \mathrm{~ms}\right)\right\}: C_{\text {operate }}\left(L_{1 R s}, \tau_{10}\right) \rightarrow C_{\text {trip }}\left(C B_{11}, \tau_{14}\right)$ \\
\hline 15 & $R_{15}\left\{\phi^{-}\left(\tau_{11}, \tau_{15}, 20 \mathrm{~ms}, 40 \mathrm{~ms}\right)\right\}: C_{\text {operate }}\left(L_{2 R s}, \tau_{11}\right) \rightarrow C_{\text {trip }}\left(C B_{12}, \tau_{15}\right)$ \\
\hline 16 & $R_{16}\left\{\phi^{-}\left(\tau_{12}, \tau_{16}, 20 \mathrm{~ms}, 40 \mathrm{~ms}\right)\right\}: C_{\text {operate }}\left(L_{4 R s}, \tau_{12}\right) \rightarrow C_{\text {trip }}\left(C B_{27}, \tau_{16}\right)$ \\
\hline 17 & $R_{17}\left\{\phi^{-}\left(\tau_{13}, \tau_{17}, 20 \mathrm{~ms}, 40 \mathrm{~ms}\right)\right\}: C_{\text {operate }}\left(L_{3 R s}, \tau_{13}\right) \rightarrow C_{\text {trip }}\left(C B_{29}, \tau_{17}\right)$ \\
\hline
\end{tabular}

\section{Declaration of competing interest}

The authors declare that they have no known competing financial interests or personal relationships that could have appeared to influence the work reported in this paper.

\section{CRediT authorship contribution statement}

Tao Wang: Conceptualization, Methodology, Investigation, Writing - original draft, Funding acquisition. Xiaoguang Wei: Validation, Formal analysis. Jun Wang: Resources, Supervision. Tao Huang: Writing
- review \& editing, Data curation. Hong Peng: Writing - review \& editing, Data curation. Xiaoxiao Song: Writing review \& editing. Luis Valencia Cabrera: Writing - review \& editing. Mario J. Pérez-Jiménez: Writing - review \& editing.

\section{Acknowledgments}

This work was partially supported by grants from the National Natural Science Foundation of China (61703345), the Key Fund Project of the Sichuan Provincial Education Department (18ZA0459), the Chunhui Project Foundation of the Education Department of China (Z201 980), the Key Scientific Research Fund Project of Xihua University (Z17108), the Open Research Subject of Key Laboratory of Fluid and Power Machinery (Xihua University), Ministry of Education (szjj201927) and the Young Scholars Reserve Talents Support Project of Xihua University. The work of the last two authors was also partially supported by the research project TIN2017-89842-P (MABICAP), co-financed by Ministerio de Economía, Industria y Competitividad (MINECO) of Spain, through the Agencia Estatal de Investigación (AEI), and by Fondo Europeo de Desarrollo Regional (FEDER) of the European Union. The authors would like to express special gratitude to Prof. G.X. Zhang for useful discussions.

\section{Appendix}

See Tables 1-8.

\section{References}

Bompard, E., Estebsari, A., Huang, T., Fulli, G., 2016. A framework for analyzing cascading failure in large interconnected power systems: A post-contingency evolution simulator. Int. J. Electr. Power 81, 12-21.

Cabarle, F.G.C., Adorna, H.N., Jiang, M., et al., 2017. Spiking neural P systems with scheduled synapses. IEEE Trans. NanoBiosci. 16 (8), 792-801.

Cardoso, G., Rolim, J.G., Zurn, H.H., 2008. Identifying the primary fault section after contingencies in bulk power systems. IEEE Trans. Power Deliv. 23 (3), 1335-1342.

Chang, C.S., Chen, J.M., Srinivasan, D., Wen, F.S., Liew, A.C., 1997. Fuzzy logic approach in power system fault section identification. IEE Proc., Gener. Transm. Distrib. 144 (5), 406-414.

Chen, W.H., 2011. Fault section estimation for power networks using logic cause-effect models. IEEE Trans. Power Deliv. 26 (2), 963-971.

Chen, W.H., 2012. Online fault diagnosis for power transmission networks using fuzzy digraph models. IEEE Trans. Power Deliv. 27 (2), 688-698. 
Chen, W.H., Tsai, S.H., Lin, H.I., 2011. Fault section estimation for power networks using logic cause-effect models. IEEE Trans. Power Deliv. 26 (2), 963-971.

Chien, C.F., Chen, S.L., Lin, Y.S., 2002. Using Bayesian network for Fault location on Distribution feeder. IEEE Trans. Power Deliv. 17 (13), 785-793.

Chin, H.C., 2003. Fault Section Diagnosis of power system using fuzzy logic. IEEE Trans. Power Syst. 18 (1), 245-250.

Díaz-Pernil, D., Cantillana, F.P., Gutiérrez-Naranjo, M.A., 2013. A parallel algorithm for skeletonizing images by using spiking neural P systems. Neurocomputing 115, 81-91.

Díaz-Pernil, D., Gutiérrez-Naranjo, M.A., 2018. Semantics of deductive databases with spiking neural P systems. Neurocomputing 272, 365-373.

Fan, W., Liao, Y., 2019. Wide area measurements based fault detection and location method for transmission lines. Prot. Contr. Mod. Power Syst. 512, 549-562.

He, Y.Y., Wang, T., Huang, K., Zhang, G.X., Pérez-Jiménez, M.J., 2015. Fault diagnosis of metro traction power systems using a probabilistic fuzzy reasoning spiking neural P system with real numbers. Rom. J. Inf. Sci. Tech. 18 (3), 256-272.

Huang, Y.C., 2002. Abductive reasoning network based diagnosis system for fault section estimation in power systems. IEEE Trans. Power Deliv. 17 (2), 369-374.

Huang, T., Voronca, S.L., Purcarea, A.A., Estebsari, A., Bompard, E., 2014. Analysis of chain of events in major historic power outages. Adv. Eletr. Comput. Eng. 14 (3), 63-70.

Huang, K., Wang, T., He, Y.Y., Zhang, G.X., Pérez-Jiménez, M.J., 2016. Temporal fuzzy reasoning spiking neural P systems with real numbers for power system fault diagnosis. J. Comput. Theor. Nanosci. 13 (6), 3804-3814.

Ionescu, M., Păun, G., Yokomori, T., 2006. Spiking neural P systems. Fund. Inform. 71 (2-3), 279-308.

Jiang, Z.Y., Li, Z.W., Wu, N.Q., Zhou, M.C., 2018. A Petri net approach to fault diagnosis and restoration for power transmission systems to avoid the output interruption of substations. IEEE Syst. J. 12 (3), 2566-2576.

Lee, H.J., Ahn, B.S., Park, Y.M., 2000. A fault diagnosis expert system for distribution substations. IEEE Trans. Power Deliv. 15 (1), 92-97.

Lin, X.N., Ke, S.H., Li, Z.T., Weng, H.L., Han, X.H., 2010. A fault diagnosis method of power systems based on improved objective function and genetic algorithm-tabu search. IEEE Trans. Power Deliv. 25 (3), 1268-1274.

Liu, W., Wang, T., Zang, T.L., Huang, Z., Wang, J., Huang, T., Wei, X.G., Li, C., 2020. A fault diagnosis method for power transmission networks based on spiking neural P systems with self-updating rules considering biological apoptosis mechanism. Complexity 2020, 2462647, 18 pages.

Luo, X., Kezunovic, M., 2008. Implementing fuzzy reasoning Petri-nets for fault section estimation. IEEE Trans. Power Syst. 23 (2), 676-685.

Ma, D.Y., Liang, Y.C., Zhao, X.S., Guan, R.C., Shi, X.H., 2013. Multi-BP expert system for fault diagnosis of power system. Eng. Appl. Artif. Intell. 26 (3), 937-944.

Mahmoudi-Nasr, P., 2019. Toward modeling alarm handling in SCADA system: a colored petri nets approach. IEEE Trans. Power Syst. 34 (6), 4525-4532.

Moghaddam, M., Chen, Q.L., Deshmukh, A.V., 2020. A neuro-inspired computational model for adaptive fault diagnosis. Expert Syst. Appl. http://dx.doi.org/10.1016/j. eswa.2019.112879.

Pan, L.Q., Păun, Gh., Zhang, G.X., Neri, F., 2017. Spiking neural P systems with communicaiton on request. Int. J. Neural Syst. 27 (8), 1750042, (13 pages).

Pan, T., Shi, X.L., Zhang, Z., Xu, F., 2018. A small universal spiking neural P system with communication on request. Neurocomputing 275, 1622-1628.

Peng, H., Wang, J., Ming, J., Shi, P., Pérez-Jiménez, M.J., Yu, W.P., Tao, C.Y., 2018. Fault diagnosis of power systems using intuitionistic fuzzy spiking neural P systems. IEEE Trans. Smart Grid 9 (5), 4777-4784.

Peng, H., Wang, J., Pérenz-Jiménez, M.J., Riscos-Núñez, A., 2019. Dynamic threshold neural P systems. Knowl. Based Syst. 163, 875-884.

Peng, H., Wang, J., Pérez-Jiménez, M.J., Wang, H., Shao, J., Wang, T., 2013. Fuzzy reasoning spiking neural p system for fault diagnosis. Inform. Sci. 235 (20), 106-116.

Prais, M., Bose, A., 1988. A topology processor that tracks network modi- fications over time. IEEE Trans. Power Syst. 3 (3), 992-998.

Păun, Gh., 2000. Computing with membranes. J. Comput. System Sci. 61 (1), 108-143.

Song, B.S., Zhang, C., Pan, L.Q., 2017. Tissue-like P systems with evolutional symport/antiport rules. Inform. Sci. 378, 177-193.

Sun, J., Qin, S.Y., Song, Y.H., 2004. Fault diagnosis of electric power systems based on fuzzy Petri nets. IEEE Trans. Power Syst. 19 (4), 2053-2059.

Tao, C.Y., Yu, W.P., Wang, J., Peng, H., Chen, K., Ming, J., 2017. Fault diagnosis of power systems based on triangular fuzzy spiking neural P systems. Commun. Comput. Inf. Sci. 618, 385-398.

Thukaram, D., Khincha, H.P., Vijaynarasimha, H.P., 2005. Artificial neural network and support vector machine approach for locating faults in radial distribution systems. IEEE Trans. Power Deliv. 20 (2), 710-721.

Tu, M., Wang, J., Peng, H., Shi, P., 2014. Application of adaptive fuzzy spiking neural P systems in fault diagnosis of power systems. Chin. J. Electron. 23 (1), 87-92.

Vázquez, E., Chacón, O.L., Altuve, H.J., 1997. An on-line expert system for fault section diagnosis in power systems. IEEE Trans. Power Syst. 12 (1), 357-362.

Wang, T., Liu, W., Zhao, J.B., Guo, X.K., Terzija, V., 2020. A rough set-based bioinspired fault diagnosis method for electrical substations. Int. J. Electr. Power 119, 105961. http://dx.doi.org/10.1016/j.ijepes.2020.105961.
Wang, J., Peng, H., 2013. Adaptive fuzzy spiking neural P systems for fuzzy inference and learning. Int. J. Comput. Math. 90 (4), 857-868.

Wang, J., Peng, H., Yu, W.P., Ming, J., Pérez-Jiménez, M.J., Tao, C.Y., Huang, X.N., 2019a. Interval-valued fuzzy spiking neural p systems for fault diagnosis of power transmission networks. Eng. Appl. Artif. Intell. 82, 102-109.

Wang, T., Wei, X.G., Huang, T., Wang, J., Peng, H., Pérez-Jiménez, M.J., ValenciaCabrera, L., 2019b. Modeling fault propagation paths in power systems: a new framework based on event SNP systems with neurotransmitter concentration. IEEE Access 7, 12798-12808.

Wang, T., Wei, X.G., Huang, T., Wang, J., Valencia-Cabrera, L., Nan, F.Z., PérezJiménez, M.J., 2019c. Cascading failures analysis considering extreme virus propagation of cyber-physical systems in smart grids. Complexity 2019, 7428458, 15 pages.

Wang, T., Zeng, S.K., Zhang, G.X., Pérez-Jiménez, M.J., Wang, J., 2015a. Fault section estimation of power systems with optimization spiking neural P systems. Rom. J. Inf. Sci. Tech. 18 (3), 240-255.

Wang, T., Zhang, G.X., Pérez-Jiménez, M.J., 2014a. Fault diagnosis models for electric locomotive systems based on fuzzy reasoning spiking neural P systems. Lecture Notes in Comput. Sci. 8961, 361-374.

Wang, T., Zhang, G.X., Pérez-Jiménez, M.J., 2015b. Fuzzy membrane computing: theory and applications. Int. J. Comput. Commun. 10 (6), 861-892.

Wang, T., Zhang, G.X., Pérez-Jiménez, M.J., 2015c. Fuzzy membrane computing: Theory and applications. Int. J. Comput. Commun. 10 (6), 861-892.

Wang, T., Zhang, G.X., Pérez-Jiménez, M.J., Cheng, J.X., 2015d. Weighted fuzzy reasoning spiking neural $\mathrm{P}$ systems: application to fault diagnosis in traction power supply systems of high-speed railways. J. Comput. Theor. Nanosci. 12 (7), 1103-1114.

Wang, T., Zhang, G.X., Rong, H.N., Pérez-Jiménez, M.J., 2014b. Application of fuzzy reasoning spiking neural P systems to fault diagnosis. Int. J. Comput. Commun. 9 (6), 786-799.

Wang, T., Zhang, G.X., Zhao, J.B., He, Z.Y., Wang, J., Pérez-Jiménez, M.J., 2015e. Fault diagnosis of electric power systems based on fuzzy reasoning spiking neural P systems. IEEE Trans. Power Syst. 30 (3), 1182-1194.

Wang, S.P., Zhao, D.M., 2019. A hierarchical power grid fault diagnosis method using multi-source information. IEEE. Trans. Smart Grid http://dx.doi.org/10.1109/TSG. 2019.2946901.

Wei, X.G., Zhao, J.B., Huang, T., Bompard, E.F., 2017. A novel cascading faults graph based transimission network vulnerability assessment method. IEEE Trans. Power Syst. 33 (3), 2995-3000.

Wen, T., Deng, Y., 2019. Identification of influencers in complex networks by local information dimensionality. Inform. Sci. 4 (4), 53-64.

Wen, T., Deng, Y., 2020. The vulnerability of communities in complex networks: An entropy approach. Reliab. Eng. Syst. Saf. 196, http://dx.doi.org/10.1016/j.ress. 2019.106782.

Wen, F.S., Han, Z.X., 1995. Fault section estimation in power systems using a genetic algorithm. Electr. Power Syst. Res. 34 (3), 165-172.

Wu, T.F., Păun, A., Zhang, Z.Q., Pan, L.Q., 2018. Spiking neuron P systems with polarizations. IEEE Trans. Neural Netw. Learn. 29 (8), 3349-3360.

Xiong, G.J., Shi, D.Y., Chen, J.F., 2013a. Implementing fuzzy reasoning spiking neural P system for fault diagnosis of power systems. In: General Meeting of the IEEE Power and Energy Society, Canada, 5970635 (5 pages).

Xiong, G.J., Shi, D.Y., Zhu, L., Duan, X.Z., 2013b. A new approach to fault diagnosis of power systems using fuzzy reasoning spiking neural P systems. Math. Probl. Eng. 2013b, 815352, 13 pages.

Yang, X.H., Chen, W.K., li, A.Y., Yang, C.S., Xie, Z.H., Dong, H.Y., 2019. BA-PNN-based methods for power transformer fault diagnosis. Adv. Eng. Inf. 39, 178-185.

Yang, J.W., He, Z.Y., Zang, T.L., 2010. Power system fault-diagnosis method based on directional weighted fuzzy Petri nets. Proc. CSEE 30 (34), 42-49.

Yu, W.P., Wang, J., Peng, H., Ming, J., Tao, C.Y., Wang, T., 2017. Fault diagnosis of power systems using fuzzy reasoning spiking neural P systems with interval-valued fuzzy numbers. Rom. J. Inf. Sci. Tech. 1 (20), 5-17.

Zeng, X.X., Zhang, X.Y., Song, T., Pan, L.Q., 2014. Spiking neural P systems with thresholds. Neural Comput. 26 (7), 1340-1361.

Zhang, Y., Chung, C.Y., Wen, F.S., Zhong, J.Y., 2016. An analytic model for fault diagnosis in power systems utilizing redundancy and temporal information of alarm messages. IEEE Trans. Power Syst. 31 (6), 4877-4886.

Zhang, X.Y., Pan, L.Q., Păun, A., 2015. On the universality of axon P systems. IEEE Trans. Neural Netw. Learn. Syst. 26 (11), 2816-2829.

Zhang, G.X., Rong, H.N., Neri, F., Pérez-Jiménez, M.J., 2014. An optimization spiking neural $\mathrm{P}$ system for approximately solving combinatorial optimization problems. Int. J. Neural Syst. 24 (5), 1440006, (16 pages).

Zhou, Q.L., Mo, H.M., Deng, Y., 2020. A new divergence measure of pythagorean fuzzy sets based on belief function and its application in medical diagnosis. Mathematics 8 (1), http://dx.doi.org/10.3390/math8010142.

Zhu, Y.L., Huo, L.M., Liu, J.L., 2006. Bayesian networks based approach for Power Systems Fault Diagnosis. IEEE Trans. Power Deliv. 21 (2), 634-639. 